\title{
A Review: Natural Superhydrophobic Surfaces and Applications
}

\author{
Mengru Jin1, Qianli Xing2, Zikang Chen ${ }^{3}$ \\ ${ }^{1}$ Ningbo Institute of Technology, Zhejiang University, Ningbo, China \\ ${ }^{2}$ China University of Petroleum (East China), Qingdao, China \\ ${ }^{3}$ Hangzhou Foreign Language School, Hangzhou, China \\ Email: jinmengrunanna@126.com,812542507@qq.com, zikang2003@foxmail
}

How to cite this paper: Jin, M.R., Xing, Q.L. and Chen, Z.K. (2020) A Review: Natural Superhydrophobic Surfaces and Applications. Journal of Biomaterials and Nanobiotechnology, 11, 110-149. https://doi.org/10.4236/jbnb.2020.112008

Received: January 4, 2020

Accepted: March 24, 2020

Published: March 27, 2020

Copyright $\odot 2020$ by author(s) and Scientific Research Publishing Inc. This work is licensed under the Creative Commons Attribution-NonCommercial International License (CC BY-NC 4.0).

http://creativecommons.org/licenses/by-nc/4.0/ (c) (i) (8) Open Access

\begin{abstract}
As the mimic biology becomes more and more important in the field of technology, superhydrophobic materials in the natural world have also become common. Superhydrophobic surfaces are used to prevent water droplets from wetting themselves which contain the micro- and nano-structures named hierarchical surfaces and exhibit the high water contact angles (WCA) that are greater than $150^{\circ}$ and perfect application foreground in both our daily lives and industry. In this work, we first discuss several surface properties and their numerical models. And then we list the surface properties of a variety of natural superhydrophobic surfaces and sum up their similarities and differences. The most recent strategies of how to apply natural superhydrophobic surfaces are also introduced within the past several years. In addition, we talk about the limitations of the current generation of superhydrophobic surfaces and prospects which looks for solutions to the problems. This review aims to enable researchers to learn more about the principles and mechanisms of superhydrophobicity and perceive the new methods for creating and modifying it.
\end{abstract}

\section{Keywords}

Superhydrophobic Surface, Wetting Models, Biomimetic, Application, Adhesion

\section{Introduction}

\subsection{Definitions}

Bionics refers to designs which are inspired by natural species. This includes adaptations of properties that are possessed by other species into the systems 
that can make convenience for humans. Bionics is a newborn subject but a far-reaching aspect of material science. Bionics, first defined by Steele, is the combination of "bio" (life) and "nics" (properties). Not until 1961, bionics began to spread and changed our life completely. Nature is always the greatest guide. Natural species usually possess some compositions or structures to help them survive. According to Natural selection theory, life has evolved more and more complex constructions in order to survive. If an inappropriate characteristic appeared on an individual species, it has a higher probability of dying. Even though there is probability that it could survive and have offspring, after all, compared to other individuals, whatever itself or its progenies, they showed a stronger tendency toward death and even were eliminated from the world. After the development of life, which could be traced back to nearly 3 billion years ago, it could be believed that the species which have survived until now have numerous beneficial properties that support them to live. These properties could also be used in human society. Therefore, bionics appeared. Although bionics has only emerged for approximately 60 years, indisputably, it has changed nearly every aspect of human life. From airplanes, to even military weapons, many of the designs of technologies around us are derived from different natural species.

Superhydrophobic surfaces are an important aspect of bionics. A superhydrophobic surface simply means that the surfaces possess the properties to repel water, causing it to bounce off its surface. According to minimum energy principle, every object has a tendency to be in the most stable state (the state which possesses the least energy). Water can form hydrogen bonds with polar molecules. But superhydrophobic surfaces usually can't form hydrogen bond with water molecules. In order to have the least energy, water molecules are more willing to form the hydrogen bond with themselves, inside the water droplet. Therefore, the water molecules nearly do not interact with the surfaces, and bead up to form nearly perfect spherical droplets. Therefore, there are many useful properties possessed by superhydrophobic surfaces such as self-cleaning or rust protection. Lotus leaves are one of the most well known examples of a natural superhydrophobic surface. Air bubbles fill in the texture under the water droplets, and the water droplets can slide around the leaf's surface without wetting [1].

\subsection{Benefits of Superhydrophobic Surfaces}

From a development perspective, over the last three decades, scientists have made many different kinds of superhydrophobic surfaces, which are beneficial for us in several aspects. First and foremost, superhydrophobic surfaces can provide the ability to protect an organism from being encumbered by water. This is commonly seen in some insects, especially those that live in the environment that has large volumes of standing or flowing water. Without superhydrophobic bodies or legs, the insects must bear the increasing load caused by adhesion of water droplets. The energy they have to expend for locomotion can increase significantly as well. This in turn can have many detrimental effects for the insect's 
survival: from the inability of escaping predators to the inability for the insect of successfully foraging.

Another major benefit is the "self-cleaning" effect. On a superhydrophobic surface, the low adhesion of water lets the droplet roll across the surface without any difficulty. Meanwhile, any contaminating particles, such as dust or debris on the surface, can be easily swept away by water's absorption or adsorption. Scientists have used this effect to create some surfaces that can stay "clean". The idea comes from the lotus leaf, which has been well-known for its ability to keep clean in any environmental condition [1].

\subsection{Scope of the Article}

In this article, we first discuss several surface properties and their numerical models, including contact angle, contact angle hysteresis, roughness, surface energy, surface tension, besides, the Cassie-Baxter and Wenzel models.

After that, we also list the surface properties of a variety of natural superhydrophobic surfaces. By analyzing their (leaves of lotus, leaves of cabbage and wings of butterflies) micro-pumps and nano-bumps, the role of surface contact angle, surface roughness and other properties can be measured as well. Finally, through summing up their similarities and differences, conclusions are carried out.

In the third part, we mainly introduce how to apply natural superhydrophobic surfaces to industrial development and daily lives. In this part, we not only provide the technology and design strategies of constructing superhydrophobic surfaces, at the same time, we but also introduce the application of artificial superhydrophobic surfaces in food, drug treatments and transportation at present.

In the end, we majorly talk about the limitation of the current generation of superhydrophobic surfaces and prospect. Introducing the defects of different surface designs, while looks for solutions to the current problems is the principal goal of this review.

\section{Theoretical Models of Contact Angle}

\subsection{Surface Properties}

\subsubsection{Surface Tension and Free Energy}

The atoms or molecules on the liquid or solid surface are to air. So they have fewer bonds with neighboring atoms or molecules compared to internal atoms or molecules. Breaking the internal chemical bonds in a liquid or solid requires a certain amount of energy. Therefore, the atoms or molecules on surface have higher energy than similar atoms and molecules in the interior. This additional energy caused by the higher energy is called surface tension or interface free energy $(\lambda)$ and measured in the energy per area units, this is $\mathrm{J} / \mathrm{m}^{2}$, or the force per unit length, which is measured in $\mathrm{N} / \mathrm{m}$ [1]. In the field of thermodynamics, there are different concepts of surface tension, surface tension force, surface energy density, and surface free energy. However, surface tension or free energy 
can be equaled when they are used in the research of superhydrophobic or superhydrophilic surfaces at a constant pressure and temperature. It's necessary to construct a ideal condition that the surface tension is equivalent to the surface energy, which require to assume there is no adsorption at the interfaces. The performance of surface energy can be summarized as follows, when a solid is in contact with liquid, the molecular attraction will reduce the energy of the system below that for the two separated surfaces. Additionally, the surface roughness can increase surface area and enhance the effect of surface energy [2].

\subsubsection{Contact Angle}

Consider a liquid drop that contacts a rigid, homogeneous, flat and inert solid surface, where the three phases of the system coexist (gas phase, liquid phase and solid phase). Simultaneously, a three phase contact line is formed (Figure 1(a)) [1]. The liquid and solid surfaces come together under equilibrium at a characteristic angle called the equilibrium contact angle $\theta_{0}$ (Figure 1(b)) [2]. The angle is a very important factor on this study, because it is used to judging hydrophobicity of a surface. According to the above definition of surface free energy, this contact angle can be determined by minimizing the net surface free energy of the system. The total energy $E_{\text {tot }}$ is given by

$$
E_{\text {tot }}=\lambda_{L A}\left(A_{L A}+A_{S L}\right)-W_{S L} A_{S L}
$$

Analyze from a geometric perspective, it is not difficult to get that

$$
\frac{d_{L A}}{d_{S L}}=\cos \theta_{0}
$$

Adhesion per unit area between the liquid and solid is expressed by the Dupré equation, which also reference theory of surface free energy

$$
W_{S L}=\lambda_{S A}+\lambda_{L A}-\lambda_{S L}
$$

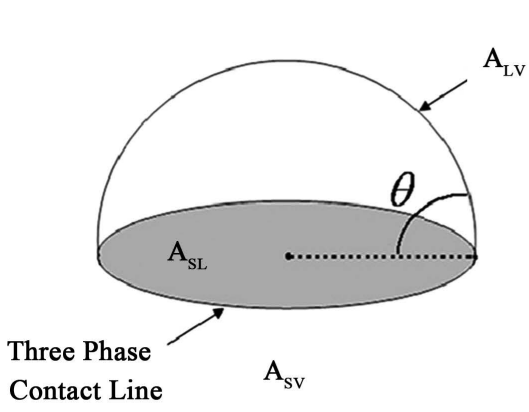

(a)

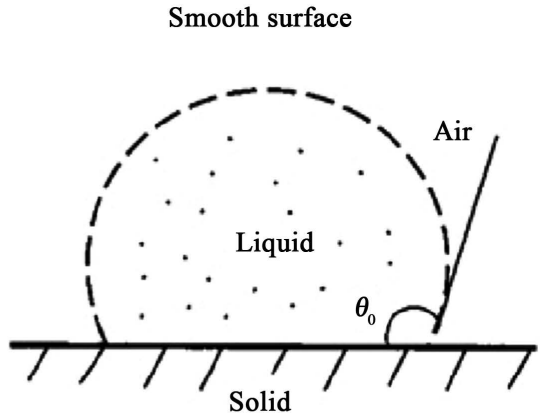

(b)

Figure 1. Contact angle representation. (a) A liquid drop showing contact angle $\theta$ balanced by three interfaces. The " $A$ " indicates contact area of interfaces. SV, SL and LV represent the interfaces between solid, liquid and vapor respectively; (b) Schematic of liquid droplet in contact with a smooth solid surface (contact angle, $\theta_{0}$ ). Image reprint from Ref [1] [2]. 
Finally, the well-known Young equation is obtained by combining the above three Equations (1), (2), and (3)

$$
\cos \theta_{0}=\frac{\lambda_{S A}-\lambda_{S L}}{\lambda_{L A}}
$$

\subsection{Wetting Models of Heterogeneous Interface}

\subsubsection{The Wenzel and Cassie-Baxter Models}

In this part, we discuss and introduce some models which simulate and calculate the contact angle of liquid on rough, heterogeneous and rigid surface.

The Young equation described the static contact angle with a homogeneous, smooth surface distinctly. Real surfaces, however, often change and vary in diverse environments, thus the contact angles cannot be explained unambiguously by the Young equation in most conditions. Because of these vast limitations, Wenzel considered a model supposing that the trenches because of the surface roughness will be penetrated and filled with the contacting liquid and proposed an equation in connection with surface roughness and surface energies in order to obtain the contact angle with complex surfaces. It is usually as:

$$
R_{f}\left(\lambda_{S V}-\lambda_{S L}\right)=\lambda_{L V} \cos \theta_{w}^{*}
$$

where $\theta_{w}^{*}$ is the Wenzel contact angle that measures the apparent contact angle on the roughness of solid surfaces. $R_{f}$ corresponds to the roughness factor, equal to the ratio of the surface area $A_{S L}$ to flat projected area $A_{F}$. So the Wenzel equation can be also written as:

$$
\cos \theta=\frac{d A_{L A}}{d A_{F}}=\frac{A_{S L}}{A_{F}} \frac{d_{L A}}{d A_{S L}}=R_{f} \cos \theta_{0}
$$

The Wenzel model indicates that the wettability can be deteriorated by the promotion of the surface roughness $R_{f}$ for a hydrophobic surface, while a hydrophilic surface might become more hydrophilic with an increase in $R_{f}$. Figure 2 shows the Wenzel interface [1].

The relationship between the static contact angle and the roughness factor is demonstrated (Figure 3) [1].

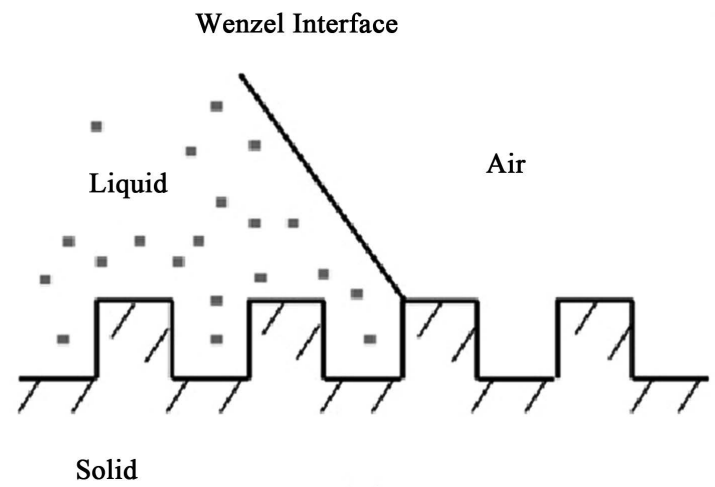

Figure 2. A droplet of which the interface is simulated by the Wenzel model placed on the rough surfaces. Image reprint from Ref [1]. 
Effect of roughness

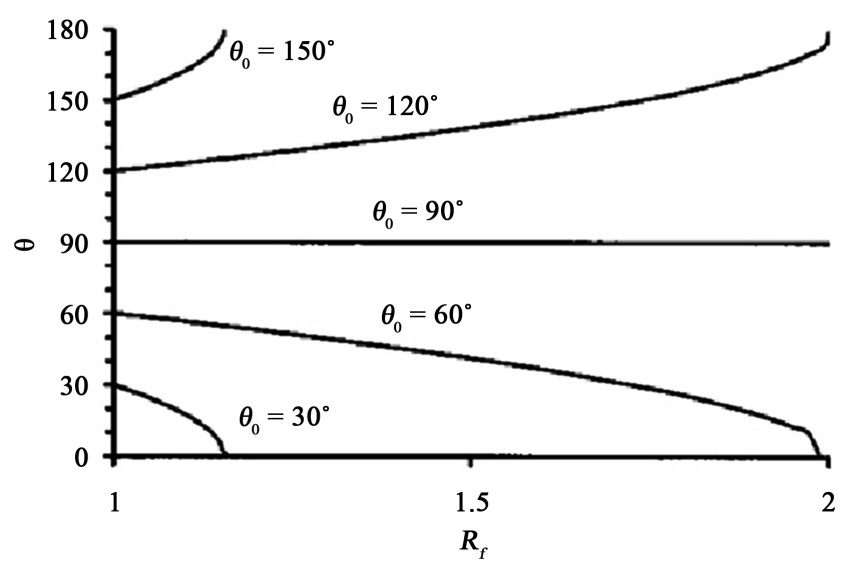

Figure 3. Contact angle for rough surface $(\theta)$ as a function of the roughness factor $\left(R_{f}\right)$ for various contact angles of the smooth surface $\left(\theta_{0}\right)$. Image reprint from Ref [1].

Besides the homogeneous wetting state, there are also two types of stable wetting states, the metastable states and the heterogeneous wetting state. So, in order to solve the problem of the heterogeneous wetting state, a surface composed of two fractions, Cassie designed the Cassie-Baxter model and used it to give a equation of the contact angle for the heterogeneous interface named the Cassie-Baxter equation which contained four parameters for two fractions, one with the fractional area $f_{1}$ and the contact angle $\theta_{1}$ and the other with $f_{1}$ and $\theta_{2}$.

$$
\cos \theta=f_{1} \cos \theta_{1}+f_{2} \cos \theta_{2}
$$

For a composite interface that includes a solid-liquid fraction and liquid-air fraction, $\theta_{2}$ would be $180^{\circ}$, and $f_{1}$ which is the ratio of the entire area of the solid-liquid interface to the total area of solid-liquid and liquid-air interfaces, would be $f_{S L}$. So the Cassie-Baxter equation can also be written as:

$$
\cos \theta=R_{f} f_{S L} \cos \theta_{0}-1+f_{S L}
$$

The formation of heterogeneous states and the relationship between the contact angle and the roughness factor and the fractional liquid-air area of hydrophilic and hydrophobic surfaces is demonstrated (Figure 4) [1].

To summarize tersely this part, we talk about two models for two different conditions. The Wenzel model is suited for a homogeneous rough surface with no air pockets and The Cassie-Baxter model is established for a heterogeneous surface, such as one containing air pockets underneath the contacting liquid. Homogeneous interface without any air pockets called the Wenzel interface, and the composite/heterogeneous interface with air pockets trapped between the rough cylinders called the Cassie-Baxter interface. However, the real state is more sophisticated. There might be a transition between homogeneous states and heterogeneous state, and in the next part, we will discuss the wetting transitions between the Wenzel and Cassie-Baxter models to solve this question. 


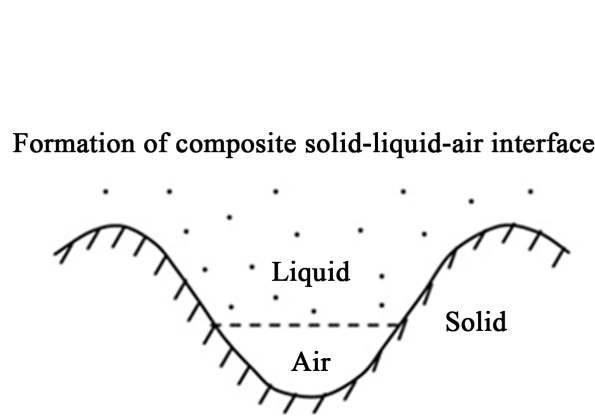

(a)

(a)

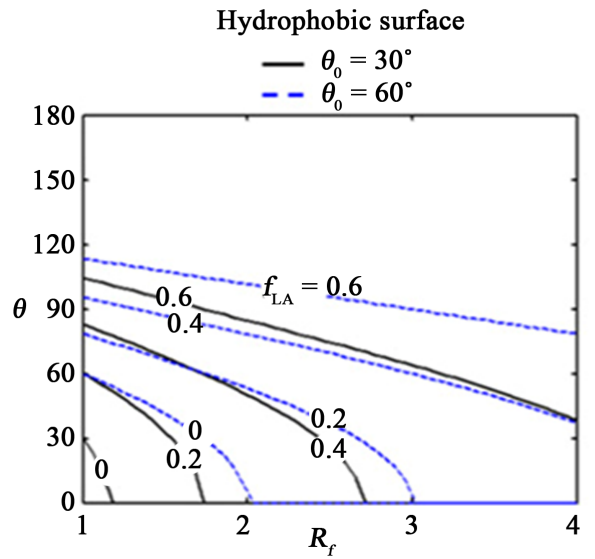

(c)

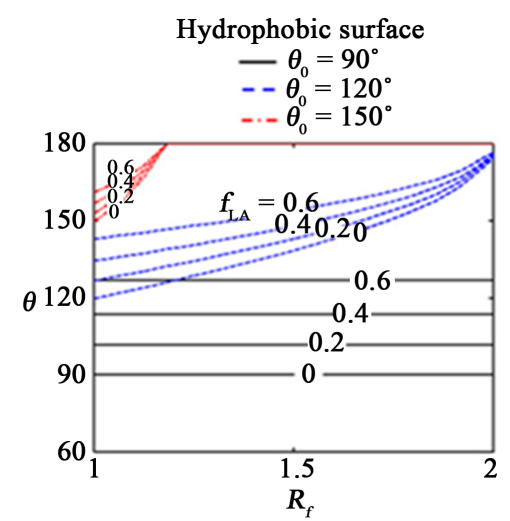

(b)

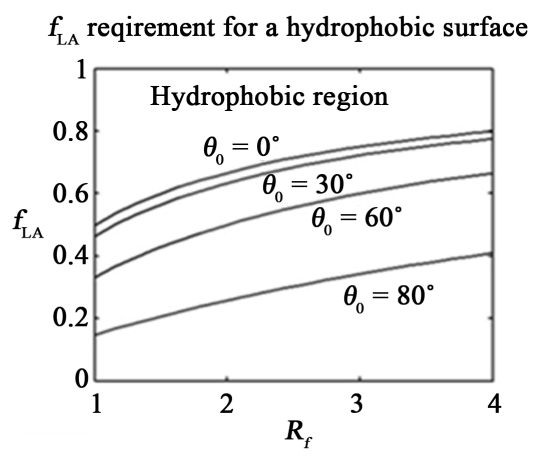

(d)

Figure 4. (a) Schematic of formation of heterogeneous state; (b) (c) A function of $R_{f}$ for different $f_{L A}$ on the hydrophobic surface and the hydrophilic surface, and (d) $f_{L A}$ requirement for a hydrophobic surface. Image reprint from Ref [1].

\subsubsection{The Hysteresis Definitions}

Tsujii and his co-workers conducted a series of experiments to test the water repellence on fractal surfaces. The resulting better condition is on a fractal surface made of an alkylketene dimer (AKD) and the contact angle is measured. In their work, the maximum contact angle reported was $174^{\circ}$, as shown in (Figure 5). They thought if there was no adsorption, this was believed that the maximum contact angle could be approaching $180^{\circ}$. But in this research, there was no clear interpretation for the contact angle hysteresis (CAH) in the experiment and theory related to the AKD superhydrophobic surfaces that mentioned before. Although there were many solid-liquid interfaces are designed with high contact angles like this after that, which have opened up a new way of thinking for the study of superhydrophobic surfaces. However, because of the contact angle hysteresis, measurement of the maximum value of contact angle lacked a certain applicability. It is worth mentioning that surfaces with roughness carefully controlled on the molecular scale it is possible to achieve contact angle hysteresis as low as $<1^{\circ}$. However, hysteresis cannot be eliminated completely, since even atomically smooth surfaces have a certain roughness and heterogeneity. 


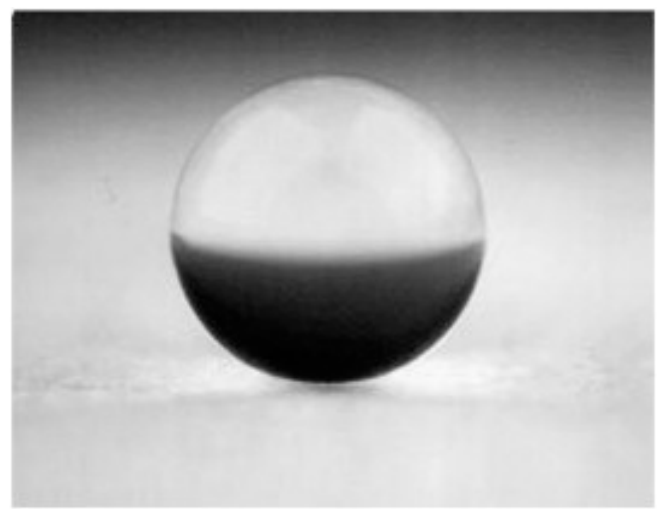

Figure 5. A water droplet of which the contact angle is $174^{\circ}$ placed on the AKD surface. Image reprinted from Ref [2].

The above example demonstrates that measurement of contact angle hyeteresis is a key point of experiments about surface superhydrophobicity. Apparently, contact angle hysteresis is another important characteristic of a solid-liquid interface. Its value is shown by measuring energy dissipated during the flow of droplets along a solid surface. Some conclusions about the relationship between contact angle hysteresis and roughness can be known from previously derived equations. The difference of cosines of the advancing and receding angles is related to the difference of those for a nominally smooth surface that are indicated $\theta_{a d v 0}$ and $\theta_{\text {reco } 0}$ in their work, Nosonovsky and Bhushan assumed that contact angle hyeteresis is equal to the adhesion hysteresis, $H_{r}$ is term and the term corresponding to effect of roughness and combine the Equation

$$
\cos \theta_{a d v}-\cos \theta_{r e c}=R_{f}\left(1-f_{L A}\right)\left(\cos \theta_{a d v 0}-\cos \theta_{r e c 0}\right)+H_{r}
$$

Then, combine Equations (8) and (9), when $f_{L A} \rightarrow 1$, that results in increasing the contact angle $(\cos \theta \rightarrow-1, \theta \rightarrow \pi)$ and decreasing the contact angle hysteresis $\left(\cos \theta_{a d v}-\cos \theta_{\text {rec }} \rightarrow 0\right)$ In the limiting case of very small solid-liquid fractional contact area under the droplet, when the contact angle is large $\left(\cos \theta \approx-1+(\pi-\theta)^{2} / 2, \sin \theta \approx \theta-\pi\right)$ and where the contact angle hysteresis is small $\left(\theta_{\text {adv }} \approx \theta \approx \theta_{\text {rec }}\right)$, based on Equations.

$$
\begin{aligned}
\pi-\theta & =\sqrt{2\left(1-f_{L A}\right)} \sqrt{R_{f} \cos \theta_{0}+1} \\
\theta_{a d v}-\theta_{r e c} & =\left(1-f_{L A}\right) R_{f} \frac{\cos \theta_{a 0}-\cos \theta_{r 0}}{-\sin \theta} \\
& =\left(\sqrt{1-f_{L A}}\right) R_{f} \frac{\cos \theta_{r 0}-\cos \theta_{a 0}}{\sqrt{2\left(R_{f} \cos \theta_{0}+1\right)}}
\end{aligned}
$$

It's all know that low contact angle hysteresis results in a very low water roll-off angle, which denotes the angle to which a surface may be tilted for roll-off of water drops (Figure 6). Low water roll-off angle is important in liquid flow applications such as in micro/nanochannels and surfaces with self-cleaning ability. For a homogeneous interface, $f_{L A}=0$, the relation about roughness and 


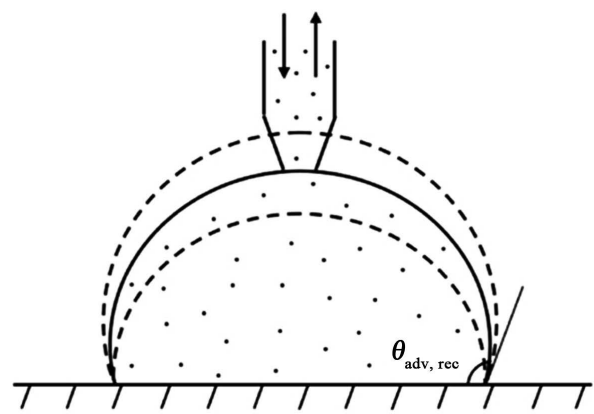

(a)

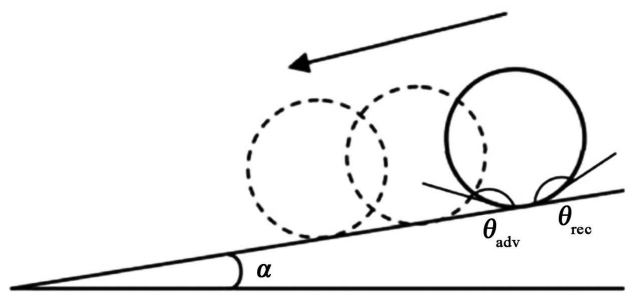

(b)

Figure 6. (a) Liquid droplet in contact with rough surface with liquid is added or removed (advancing and receding contact angles are $\theta_{a d v}$ and $\theta_{\text {rec }}$, respectively) and (b) tilted surface profile (tilt angle, a with a moving liquid droplet). Image reprint from Ref $[2]$.

contact angle hysteresis can be given by observing Equations (10) and (11). Generally, as the roughness increases (high $R_{f}$ ), the hysteresis angle increases (high values of $\theta_{a d v}-\theta_{\text {rec }}$ ). For composite interface that $f_{L A}$ is a non-zero number, an approach to unity of the $f_{L A}$ provides both high contact angle and small contact angle hysteresis. Therefore, the composite interface has self-cleaning features that worth developing [2].

\subsubsection{Wetting Transitions between the Wenzel and Cassie-Baxter Models}

As well known, a stable composite interface is very important premise for the Cassie-Baxter model and the Wenzel model, nevertheless it is also extremely hard to keep it. So, to study and understand model of transition from the Wenzel model to the Cassie-Baxter model is critical. Nosonovsky and Bhushan suggested that despite the transition can be in progress by some ways, for instance, put pressure on the droplet, it is not a reversible event, which is difficult to return. "the Cassie-Baxter transition", the name of the transition, happens since the net surface energy of both the Wenzel state and the Cassie-Baxter state develops into equal which means that the contact angle measured by the Cassie-Baxter model is the same as the contact angle measured by the Wenzel model. The opinion of Extrand is that the weight of the droplet is related closely to the transition and put forward the contact line density model, if the weight out striped the surface tension at the triple line, the transition will take place. Although there are many scholars studying it and plenty of experiments proving a lot of results, it is still not clear. Critical contact angle is a considerable concept 
about the transition. Contact angle of a rough surface as a function of surface roughness parameter is expressed (Figure 7) [1] [2].

It is clear from Figure 7 that an increasing of roughness might make the transition between the Wenzel and Cassie-Baxter regimes at the intersection point, the critical contact angle. So we can get:

$$
\cos \theta_{c}^{\prime}=\frac{1-\phi_{s}}{r_{f} \cdot \phi_{s}-r}
$$

where $\theta_{c}$ is the critical contact angle.

It means that when the Young contact angle is higher than $\theta_{c}$, the heterogeneous states will be favoured, on the contrary, the homogeneous states will be favoured. However, the critical point is not the unique factor which influences the transition. Many experiments show that, although the Young contact angle is lower than $\theta_{c}$, the transition does not necessarily appear because of the energy barriers, so the droplets might prefer to remain in the metastable Cassie-Baxter state.

\section{Several Different Types of Natural Superhydrophobic Surfaces}

\subsection{The Properties of Natural Superhydrophobic}

\subsubsection{Leaves of Lotus}

The surface of the lotus leaf is a well-known superhydrophobic surface. Another important ability that benefits from the excellent superhydrophobic is self-cleaning, which was named "Lotus effect". In recent years, scanning electron microscopy was widely used to reveal the microstructure of the lotus leaf surface to explore the source of its superhydrophobic properties. Some phenomena can be observed under SEM. The lower epidermis consists of convex cells covered with wax tubules and contains only few stomata. The upper epidermis consists of papillae with a dense coating of agglomerated wax tubules. It is now understood that this unique hierarchical structure is responsible for the superhydrophobic properties of the lotus leaf (Figure 8) [3].
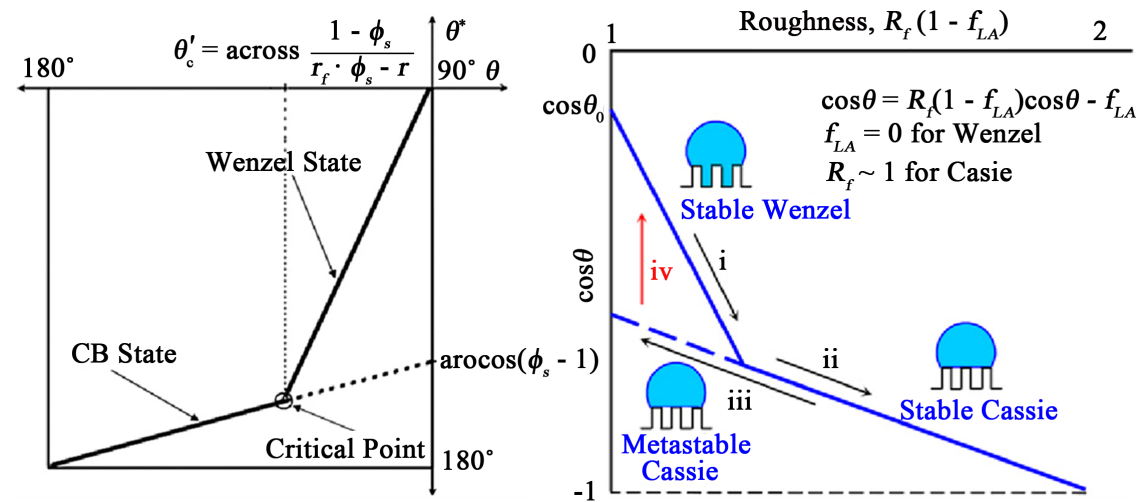

Figure 7. Wenzel and Cassie-Baxter models with impact from $R_{f}$ Image reprint from Ref [1] [2]. 

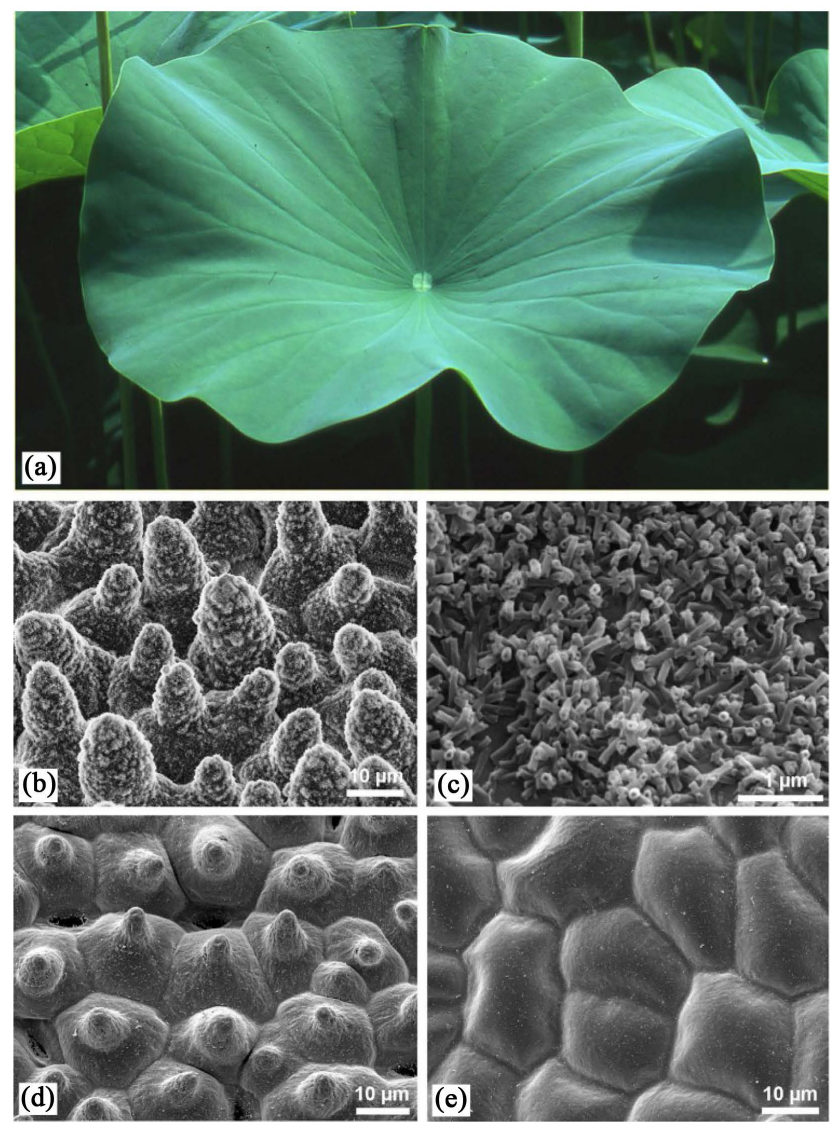

Figure 8. (a) Lotus leaves, which exhibit extraordinary water repellency on their upper side; (b) Scanning electron microscopy (SEM) image of the upper leaf side prepared by "glycerol substitution" shows the hierarchical surface structure consisting of papillae, wax clusters and wax tubules; (c) Wax tubules on the upper leaf side; (d) Upper leaf side after critical-point (CP) drying. The wax tubules are dissolved, thus the stomata are more visible. Tilt angle $15^{\circ}$; (e) Leaf underside (CP dried) shows convex cells without stomata. Image reprinted from Ref [3].

According to the Cassie-Baxter model presented in the previous section, the hierarchical structure can be known to cause superhydrophobicity, that is the contact angle will be greater than $150^{\circ}$. The superhydrophobicity of the lotus leaf surface can be demonstrated by the Cassie-Baxter model. In contrast, the hydrophobicity of the rose petal surface is more in line with the Wenzel model (Figure 9). The surface of the lotus leaf is the natural surface that is currently measured with the largest contact angle $\left(\theta=162^{\circ}\right)$. However, some species with a dense layer of epicuticular wax crystals but epidermal cells are flat also have a large contact angle, and some are even measured more than $160^{\circ}$. Therefore, it's not difficult to see that the superhydrophobic sample cannot be distinguished only by the size of the contact angle and contact angle hysteresis or roll-off (tilt) angles show a clearer the difference between species. It became obvious that the outstanding and stable superhydrophobicity of the lotus leaf relies on the combination of optimized features such as the surface topography, robustness and the unique properties of the epicuticular wax [1] [3]. 


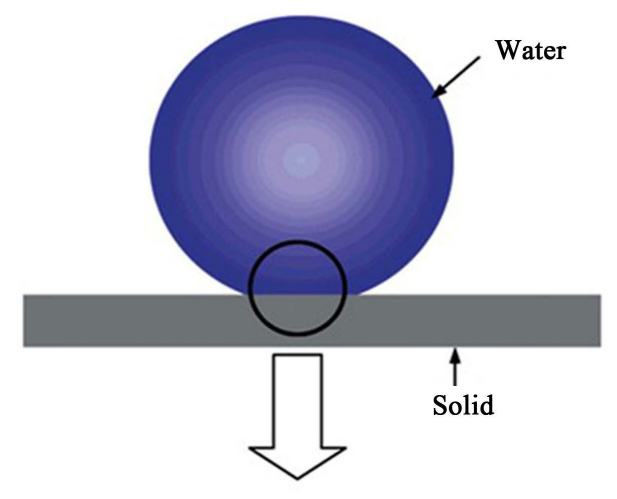

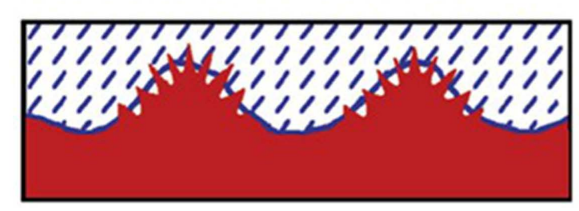

Petal (Cassie impregnating wetting state)

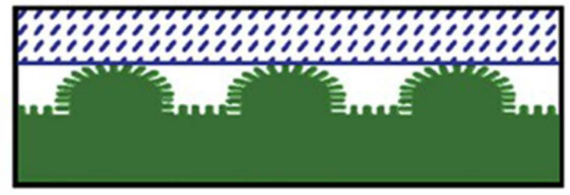

Lotus (Cassie's state)

Figure 9. Schematic illustrations of a drop of water in contact with the petal of a red rose (the Cassie impregnating wetting state) and a lotus leaf (the Cassie's state). Image reprinted from Ref [2].

Compared with other species from the surface topography, lotus has the highest density of papillae with varying heights and the smallest diameter of the papillae. Other species not only have larger mastoid diameters, but also cover different types of waxes: wax platelets (E. myrsinites and C. esculenta) and a wax film (A. macrorrhiza) which covers cuticular foldings (Figure 10). The diameter of the lotus papillae is much smaller, which reduces the area of contact with water droplets. Strictly, the contact area between the surface and the water is not uniform, but the minimum contact area is the root cause of the very low water adhesion, and further, the roll-off angle of the lotus leaf is also small.

AFM scan data was used as the approximation of the roughness factor for the leaves on the micro- and nanoscale. In Table 1, roughness factors for several different leaves are presented. Using roughness factor values, along with the contact angles $(\theta)$ from both superhydrophobic and hydrophilic surfaces, $153^{\circ}$ and $152^{\circ}$ in lotus and Colocasia, and $76^{\circ}$ and $84^{\circ}$ Fagus and Magnolia, respectively. The Wenzel equation (Equation (10)) be used to calculate the contact angle $\left(\theta_{0}\right)$ of a smooth surface for microbumps; The Cassie-Baxter equation (Equation (12)) be used to calculate for the nanobump. Consider the influence factors of roughness $\left(R_{f}\right)$ in calculating the contact angle contact angle $(\theta)$ and it's recorded Table 1. At the nanoscale, the roughness factor and the difference $(\Delta \theta)$ between $\theta$ and $\theta_{0}$ are higher than those in micron level, which means that the nanobumps on top of the microbumps increase the contact angle more effectively than the microbumps. However, for hydrophilic leaves, the change of values of andare varies little on both scales. The difference between the microbumps and the nanobumps used to increase the surface hydrophobicity is 


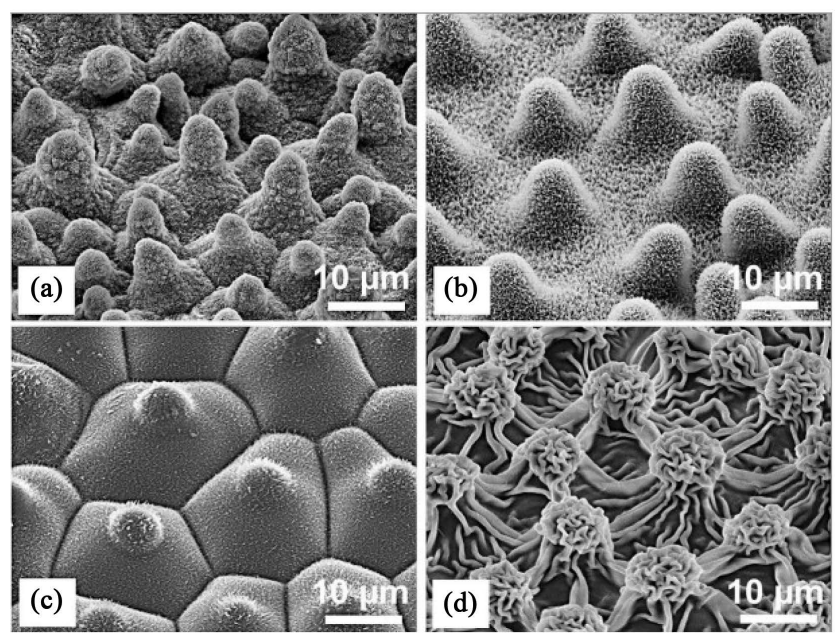

Figure 10. SEM images of the papillose leaf surfaces of Nelumbo nucifera (Lotus) (a); Euphorbia myrsinites (b); Colocasia esculenta (c); and Alocasia macrorrhiza (d). Image reprint from Ref [2].

Table 1. Roughness factor and contact angle $\left(\Delta \theta=\theta-\theta_{0}\right)$ calculated using $R_{f}$ on the smooth surface for hydrophobic and hydrophilic leaves measured using an AFM, both microscale and nanoscale.

\begin{tabular}{ccccc}
\hline Leaf $($ contact angle $)$ & Scan size & State & $R_{f}$ & $\Delta \theta(\mathrm{deg})$ \\
\hline Lotus $\left(153^{\circ}\right)$ & $50 \mu \mathrm{m}$ & Dried & 5.6 & $54^{*}$ \\
& $2 \mu \mathrm{m}$ & Fresh & 20 & $61^{* *}$ \\
& $50 \mu \mathrm{m}$ & Dried & 16 & $60^{* *}$ \\
Colocasia $\left(152^{\circ}\right)$ & $2 \mu \mathrm{m}$ & Dried & 8.4 & $56^{*}$ \\
& bump & Dried & 18 & $60^{* *}$ \\
& $2 \mu \mathrm{m} \mathrm{ridge}$ & Fresh & 18 & $59^{* *}$ \\
Fagus $\left(76^{\circ}\right)$ & $50 \mu \mathrm{m}$ & Dried & 15 & $60^{* *}$ \\
& $2 \mu \mathrm{m}$ & Fresh & 3.4 & $59^{* *}$ \\
Magnolia $\left(84^{\circ}\right)$ & $50 \mu \mathrm{m}$ & Fresh & 5.3 & $-10^{*}$ \\
& $2 \mu \mathrm{m}$ & Fresh & 3.8 & $-2^{* *}$ \\
& & 3.6 & $14^{* *}$ \\
\hline
\end{tabular}

${ }^{*}$ Calculations made using Wenzel equation. ${ }^{* *}$ Calculations made using Cassie-Baxter equation. We assume that the contact area between the droplet and air is the half of the whole area of the rough surface. Table from Ref [1].

the effect on the contact angle hysteresis. In other words, the ease with which the water droplets roll on the surface. Air pockets below the droplets result in reduced contact with the solid surface, reduced contact angle hysteresis, and increased contact angle. Surfaces with nanobumps have a higher roughness factor than microbumps. As the distance between the microbumps becomes larger, the possibility of cavitation formation decreases, and a high contact angle hysteresis is caused. Therefore, on the surface having nanobumps, the contact angle is high 
and the contact angle hysteresis is low, and the droplets are easily rebounded and can roll. Natural hydrophobic and self-cleaning surfaces, such as lotus leaves, are a good example of this phenomenon [1].

Wax tubules of lotus is an indispensable condition that make the surface superhydrophobic. Both the upper side and the lower side of the lotus leaf are covered with wax tubules. However, these wax tubules have different shapes on two sides of the lotus leaf, which be shown on the SEM images (Figure 11(a) and Figure 11(b)). In the lower side, the wax tubules are longer ( 1 to $2 \mu \mathrm{m})$ and thicker (ca. $150 \mathrm{~nm}$ ). The tiny tubes like these have been found to frequently occur on many plants species and that are typical "nonacosanol" tubules. In contrast, in the upper leaf side, the wax tubules are very short $(0.3-1 \mu \mathrm{m})$ and thin $(80-120 \mathrm{~nm})$ but very dense compare with the wax tubles on the lower side. Figure 11 shows on a clearly arranged area, approximately 200 tubules per $10 \mu \mathrm{m}^{2}$ on the upper side, but only about 63 tubules per $10 \mu \mathrm{m}^{2}$ on the lower side of the same leaf. The space is very tight space between the wax tubules on the upper side of the lotus leaf, which is much smaller than that of other wax crystals such as platelets (Figure 11(c) and Figure 11(d)) and other tubular waxes (Figures 11(b)-(f)). It is obvious that the droplets such as water are difficult to wet the surface of the lotus leaf. Because these distances between the wax crystals

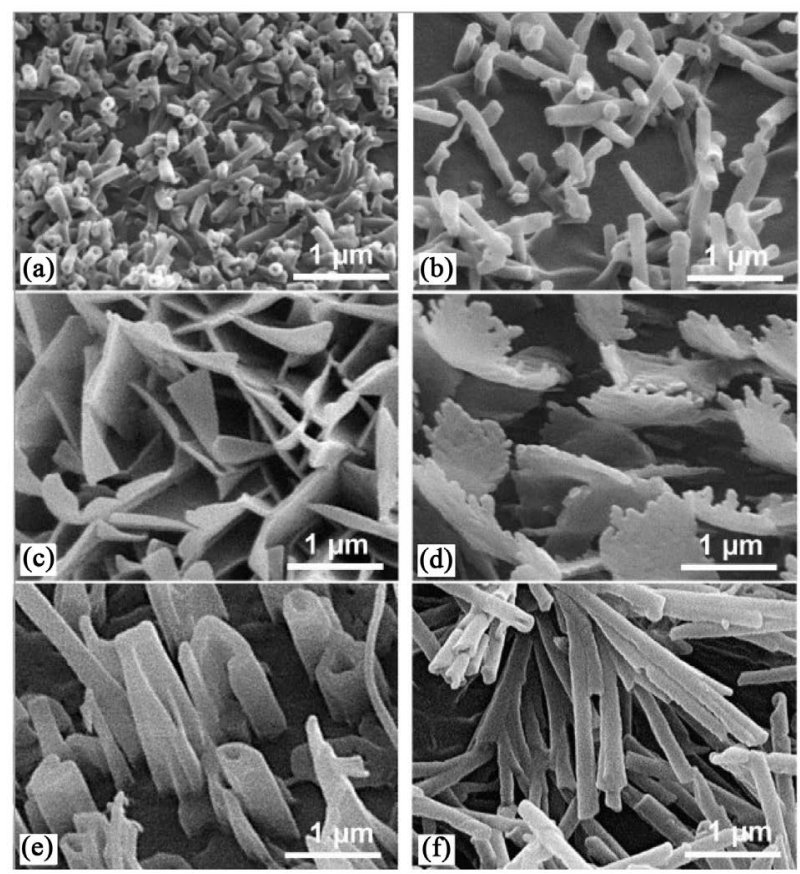

Figure 11. Epicuticular wax crystals in an area of $4 \times 3 \mu \mathrm{m}^{2}$. The upper side of the lotus leaf (a) has the highest crystal density (number per area) of wax crystals and the smallest spacings between them. Lotus upper side (a) ca. 200 tubules per $10 \mu \mathrm{m}^{2}$; (b) Lotus underside ca. 63 tubules per $10 \mu \mathrm{m}^{2}$; (c) Euphorbia myrsinites ca. 50 platelets per $10 \mu \mathrm{m}^{2}$; (d) Yucca filamentosa ca. 17 platelets with over 80 jags per $10 \mu \mathrm{m}^{2}$; (e) Brassica oleracea ca. 22 rodlets and tubules, and (f) Eucalyptus macrocarpa ca. 50 tubules per $10 \mu \mathrm{m}^{2}$. The larger spacing between the wax crystals of the other surfaces compared to the lotus upper side is obvious. Image from Ref [3]. 
determine the pressure (capillary pressure), and the penetration of water droplets. The distance between the wax tubes on the upper side of the lotus leaf is very small and the arrangement is quite dense, so it is difficult for water to penetrate the air part below to wet the surface [3].

These three characteristics are the main reasons why the lotus leaf exhibits outstanding superhydrophobic and self-clean properties. As its internal structure is continuously explored, it has become the object of reference for many scientists when designing super-hydrophobic surfaces. Hence, so-called lotus effect also has far-reaching influence in the field.

\subsubsection{Wings of Butterflies}

The surface of butterflies' wings is a specific example of the natural superhydrophobic surface in insects. There are four general cuticular surface: layered cuticles, fractal structures, denticles and setae [4].

The surface of butterflies' wings is considered to be a layered cuticle. The wings of these insects have very high water contact angles (greater than or equal to $150^{\circ}$ ), Figure 12 shows that their structures [4].

The wings are covered in a lot of overlapping micro-sized (typically $10 \mu \mathrm{m}$ $100 \mu \mathrm{m})$ scales lying in one direction, with the structure of each scale consisting of parallel ridges running along the long axis of the scale connected by horizontal links and covered with multiple nano-sized protuberances (Figure 13).

Besides exhibiting superhydrophobicity, the structure produces anisotropic wetting characteristics in that water droplets preferentially roll off of the wing in one direction. According to some researches, the contact angles of wings' surface of 49 kinds of butterflies have been measured, list in Table 2 [4].

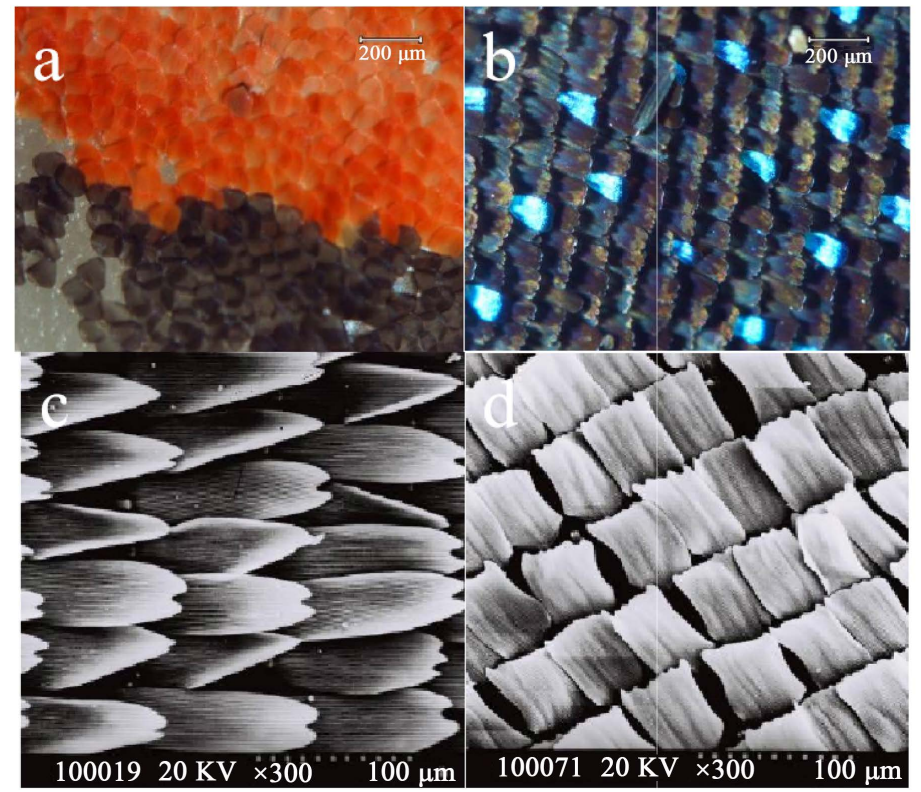

Figure 12. Somatic microscopic photograph of butterfly wing scales and the scales of butterfly wings are imbricate, (a) Parnassius bremeri; (b) Papilio maackii; (c) Papilio machaon; (d) Everes argiades. Image reprinted from Ref [4]. 


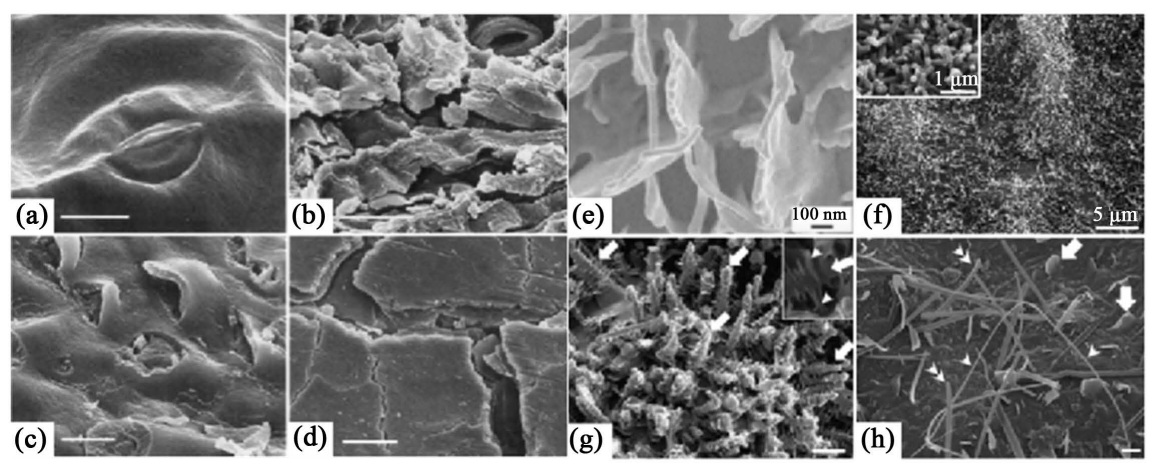

Figure 13. Major nanoscale morphology of plant cuticular waxes. Panels (a)-(d) represent (a) the fifilm (scale bar $=10 \mu \mathrm{m}$ ); (b) crust (scale bar $=20 \mu \mathrm{m}$ ); (c) smooth layer (scale bar $=10 \mu \mathrm{m})$ and $(\mathrm{d})$ the fifissured layer (scale bar $=20 \mu \mathrm{m}$ ) morphologies, respectively. Panels; (e)-(h) depict the major wax crystal morphologies, in the form of (e) platelets, (f) tubules; (g) rodlets (transversely ridged rodlets show, scale bar $=2 \mu \mathrm{m}$ ) and (h) threads (scale bar $=1 \mu \mathrm{m})$. Image reprinted from Ref [4].

Table 2. Structural parameters and contact Angle of butterfly wing surface scales. Table reprint from Ref [4].

\begin{tabular}{cccccc}
\hline \multirow{2}{*}{ Butterfly species } & \multicolumn{3}{c}{ Structural parameters } & \multicolumn{2}{c}{ Contact angle $\left(^{\circ}\right)$} \\
\cline { 2 - 6 } & Scale size & Distance & Protrusion high & No phosphorus & Phosphorus \\
\hline Argynnis paphia & $70 * 35$ & 54 & 0.34 & 123.0 & 156.6 \\
Limenitis populi & $90 * 50$ & 76 & 0.20 & 129.0 & 150.3 \\
Pontia daplidice & $80 * 60$ & 74 & 0.50 & 112.1 & 139.3 \\
\hline
\end{tabular}

It is not difficult to see that all the contact angles are bigger than $150^{\circ}$, which means that these surfaces are superhydrophobic. The scales on those wings are too small, so the distance between the scales undertakes a little bit more contact area when the droplet contacts with the surface, which increases the hydrophobility. After the scales were moved, those contact angles were smaller (Figure $14)$.

\subsubsection{Petal of Red Rose}

Red rose petal is another surface that with very high water contact angles. Unlike many superhydrophobic surfaces, the Wenzel state was used to describe a drop of water in contact with the petal of a red rose, and more precisely, the Cassie impregnating wetting state (Figure 15). By observing the microstructure or nanostructure of the organism, some explanations about its unique properties can be obtained. There are SEM images of the surface of a red rose petal (Figure 15(a) and Figure 15(b)) and the micropapillae first time was shown that has a closely arrayed pattern of red rose petal surface. These densely packed papillae and nanofolds on the top of the papillae increase the surface roughness at a perspective of microscopic. However, the surface of red rose petal has amazingly high adhesion with water, which indicate the phenomenon that water won't roll off even if the petals are turned upside down when the droplets appear spherical 

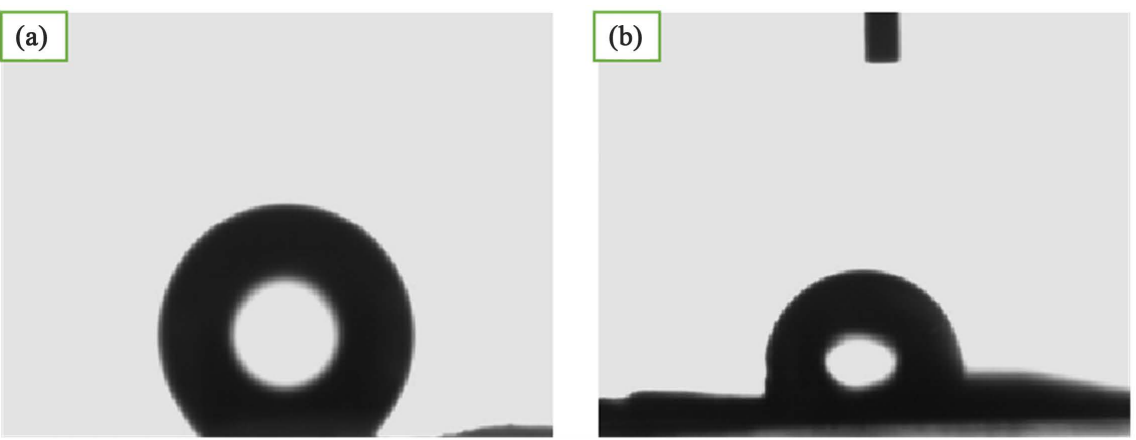

Figure 14. (a) The angle of contact when the wing of Argyronome laodice butterfly has scales; (b) The contact Angle of the wing of Argyronome laodice butterfly without scales. Image reprinted from Ref [4].

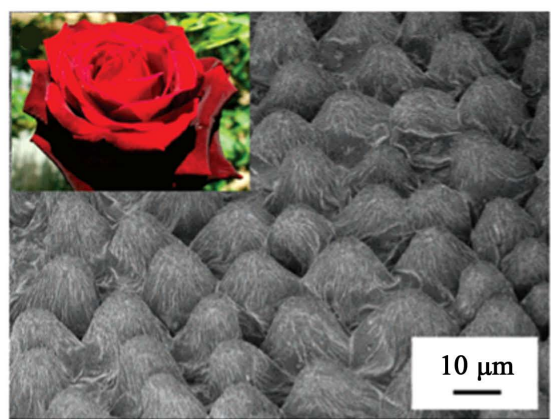

(a)

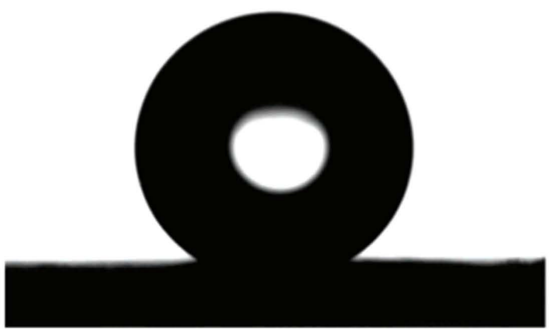

(c)

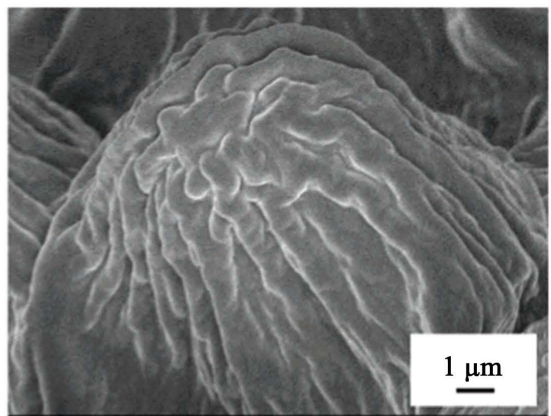

(b)

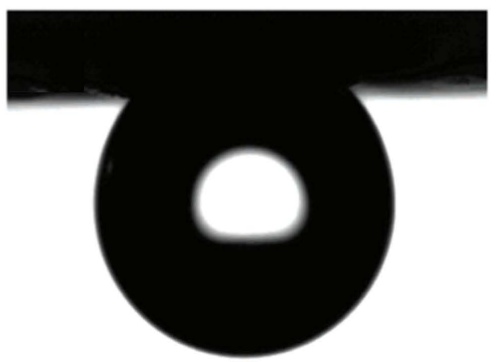

(d)

Figure 15. (a) (b) SEM images of the surface of a red rose petal, showing a periodic array of micropapillae and nanofolds on each papillae top; (c) Shape of a water droplet on the petal's surface, indicating its superhydrophobicity with a contact angle of $152.4^{\circ}$; (d) Shape of water on the petal's surface when it is turned upside down. Image reprint form [5].

on the surface of rose petals that is in superhydrophobic state (contact angle $\theta=152.4^{\circ}$ ) (Figure 15(c) and Figure 15(d)) [5]. Interestingly, there are special surfaces in nature that also have this phenomenon, typically, such as the gecko's sole. In order to distinguish from the "lotus effect", the phenomenon was defined as "petal effect".

So what makes the red rose petals have high adhesion? The Cassie impregnating wetting state mentioned above is the key. The surface of the red rose petal is composed of closely arranged papillae and nanofolds on the top. When the water 
drops on the surface, it only wets the periphery of the micron-sized papillae and does not enter those nanofolds and the hypothesis can be visually represented in Figure 9. In the Cassie impregnating wetting regime, water droplets enter into the "large" grooves of the petal but not into the "small" ones. This indicates why small water drops sealed in micropapillae would be clinched to the petal's surface, showing a high contact angle hysteresis even if the surface was turned upside down.

Furthermore, experiments were developed to prove that the hypothesis is convincing in the previous work. Fresh and dry rose petals are used as samples for comparison in experiments and images of SEM are shown in Figure 16(a) and Figure 16(b). The surface of the fresh rose consists of a full-bodied micropapillae with a radius of $20 \mu \mathrm{m}$ and the nanofolds on the top are clearly visible. While the micropapillae structure on the surface of the dry rose has shrunk, but there are still nanofolds on the top. Then the curve of the force between the water droplets and the surface of the different rose petal samples during the process of contact with the water droplets to the water droplets is made. As can be seen from Figure 16(c), the fresh rose has a very large adhesion on the front, about $110 \mu \mathrm{N}$. It can be seen from Figure 16(d) that the adhesion of the front of the dry rose is significantly reduced compared to the fresh rose front, which is only about $70 \mu \mathrm{N}$. Combine the above study of the microscopic surface of roses, after the rose petals are dry, the microscopic morphology of the micropapillae changes significantly, the main reason is micropapillae atrophy. The overall structure of micropapillae becomes smaller and the spacing between them are
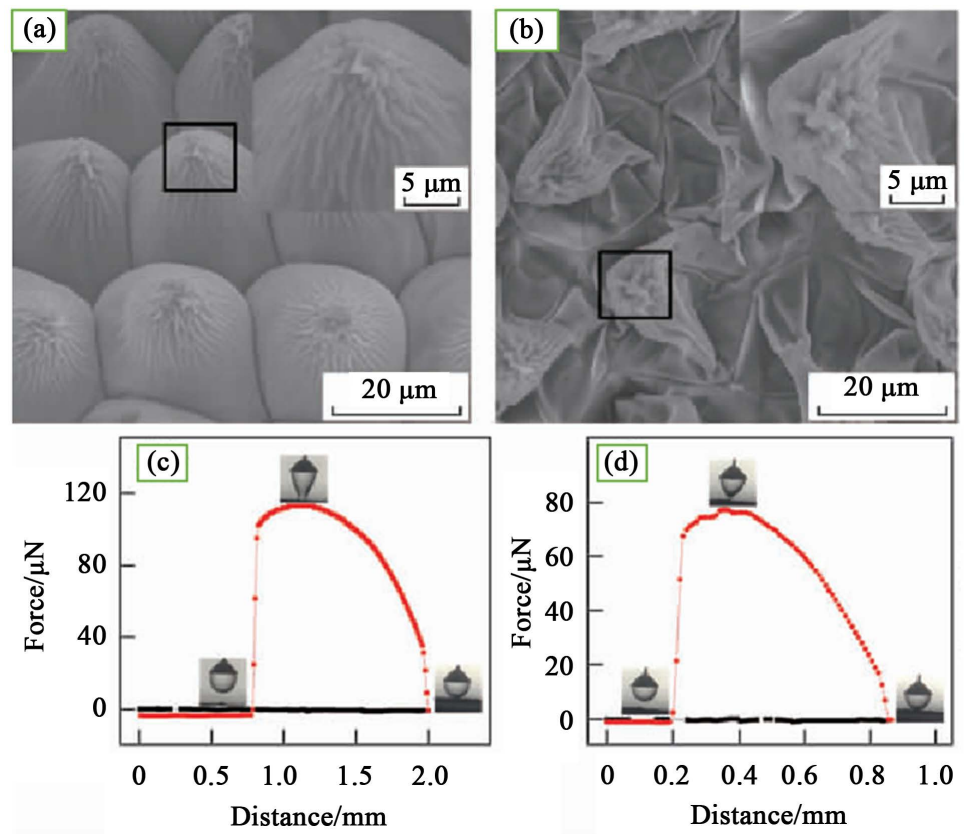

Figure 16. SEM images of rose petal surface. (a) Obverse surface of fresh rose; (b) Obverse surface of dry rose. Curves of force-distance recorded before and after the water droplet contacts with red rose petals. (c) Obverse surface of fresh rose; (d) Obverse surface of dry rose. Image reprint from [6]. 
increase. Additionally, another important point is that the top of the micropapillae also shrinks significantly. Due to the increase of the mastoids, the water droplets are more easily immersed in the microstructure, the contact angle becomes smaller, but the adhesion increases. However, and the atrophy of the nanofolds at the top of the micropapillae reduces the contact area between the water droplets and the surface of the petal. As a result, the adhesion of the petal surface is reduced, while verifying the above hypothesis, it also indicates that the nanostructures have a more significant influence on the adhesion force in the hierarchical structure of the petal surface [6].

\subsection{Commonality of Natural Superhydrophobic Surfaces}

The lotus leaf is a superhydrophobic surface, which means that any water that spills on it will bounce of the surface, since it has a Cassie-Baxter state of surface and mushroom-shape bumps. While the rose petal is a special case, it can hold the water on the surface from wetting the petal and keep them in place; and this is because that rose petals have a Cassie impregnating wetting state, which means that the petals contain hierarchical structures in multiple different scales which function differently so it kept the water droplets in place. The butterfly wings, in the case, have a directional adhesion superhydrophobic surface; this structure helps the butterfly keep the water droplets away from its body, since the stripe structure allows the water droplet to flow off the wings. Although these textures are different but there are still similarities: 1) they share the Cassie-Baxter structure, 2) they are both superhydrophobic surface in some scale, 3) they both have hierarchical structures in different scales.

\subsection{Special Properties of Different Natural Superhydrophobic Surface}

There are many superhydrophobic surfaces in nature, such as the three introduced above: lotus leaves, butterfly wings, red rose petals. There is obviously a commonality between them, that is, they are all in a superhydrophobic state. However, each superhydrophobic surface has its own special properties that different from others. In order to understand the unique characteristics of these superhydrophobic surfaces, the first step is to explore the difference in microstructure of surface, which is the main source of special properties for these superhydrophobic surfaces. SEM images show microstructure of these surfaces in Figure 17. The upper side of the lotus leaf consists of a mass of closely arranged micropapillae, which are covered by a nanoscale wax tube (Figure 17(a) and Figure 17(b)). The wings of the butterfly are covered by a large number of closely spaced square scales and overlap each other to form a periodic hierarchy. Further magnified views show many individual undulating stripes on each microscale (Figure 17(c) and Figure 17(d)). It is worth mentioning that these ordered stripes show a uniform slope on each microunit, and the direction along these stripes is called the radial outward (RO) direction [7]. (Figure 17(e) and Figure 17(f)) show the microstructure of red rose petal. Similar to the lotus 


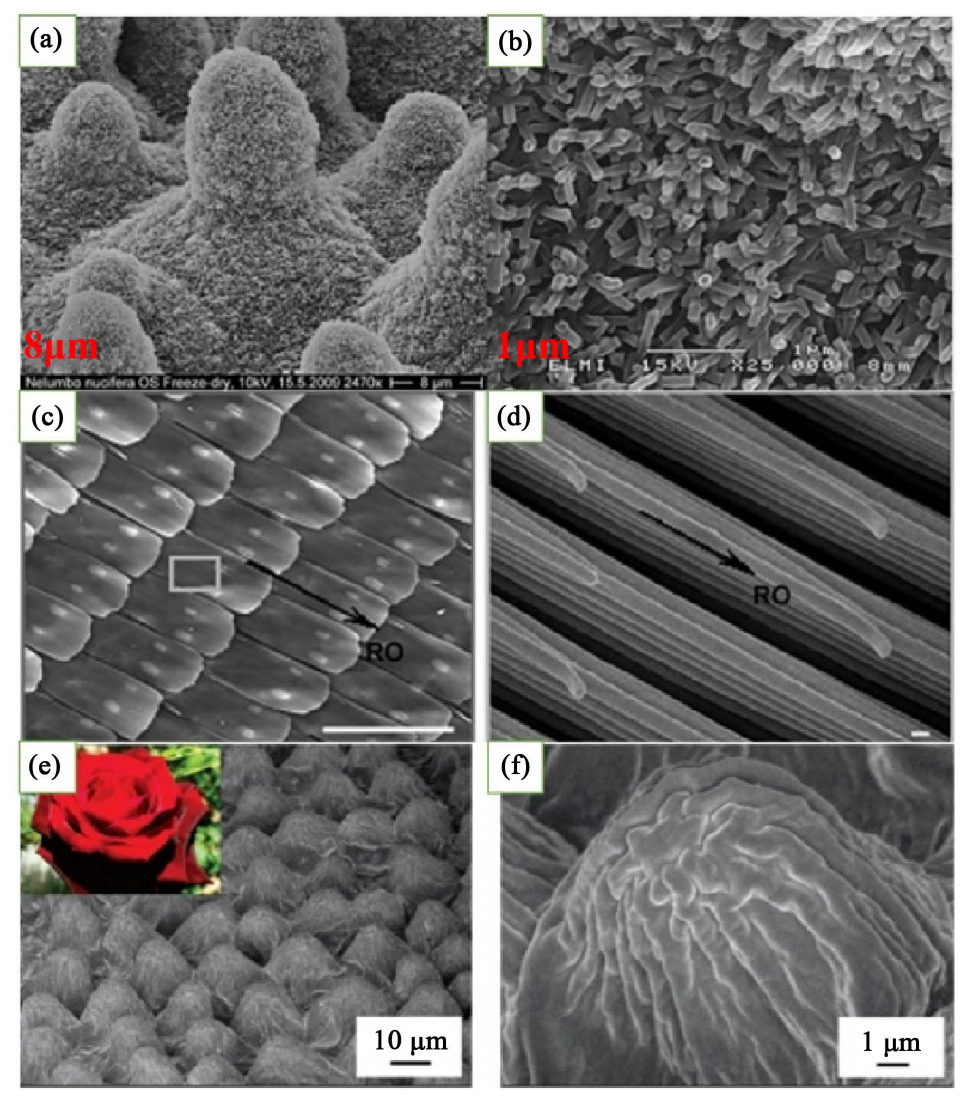

Figure 17. (a) The upper side surface of the lotus leaf without the shrinkage artifact. Scale bar $=8 \mu \mathrm{m}$; (b) The wax tubules from the upper side of the lotus leaf. Scale bar $=1 \mu \mathrm{m}$; Hierarchical micro- and nanostructures on the surface of the wings. Image reprint from Ref [2]; (c) (d) SEM images of the periodic arrangement of overlapping micro-scales on the wings and fine lamella-stacking nano-stripes on the scales. Scale bars: (c) $100 \mathrm{~mm}$; (d) $100 \mathrm{~nm}$. Image reprint from Ref [7]; (e) (f) SEM images of the surface of a red rose petal, showing a periodic array of micropapillae and nanofolds on each papillae top. Image reprint from Ref [5].

surface, the petal surface also consists of closely arranged micropapillae, except that the papillae of the petal are flatter than the lotus leaf. Many of the nanofolds on the top of the micropapillae are characteristic of the rose petal.

These structural differences lead to differences in the properties of these natural superhydrophobic surfaces, the most important of which is the adhesion to water droplets. There are many wax tubules and nanobumps on the surface of the lotus leaf. This is a typical superhydrophobic surface structure that can be explained by the Cassie-Baxer model. Excellent super-hydrophobic structure on the surface of the lotus leaf provides it with a large contact angle $\left(\theta>160^{\circ}\right)$ and a very small roll-off angle $\left(\theta<5^{\circ}\right)$ (Figure 18(a) and Figure 18(b)). On butterfly wings, water droplets deposited on the wing exhibit a unique precipitation behavior that is roll off in the RO direction and against the RO direction by tilting the wing downwards or upwards, respectively. Figure 18(c) shows a representative image of the process of rolling off the surface of the wing as the three droplets are gradually tilted downward. It can be seen that as the wing is tilted 

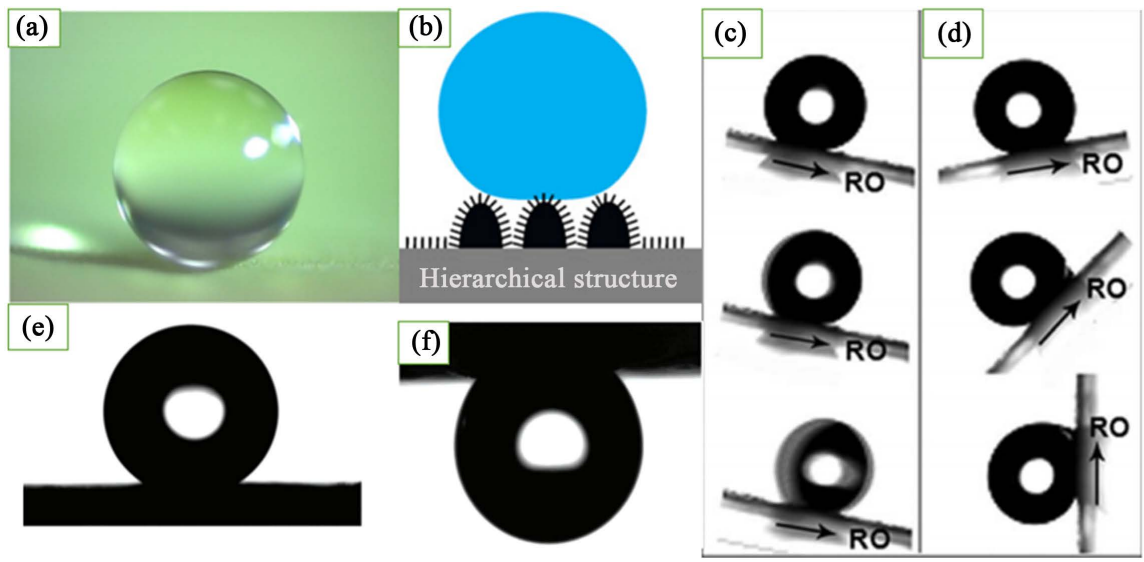

Figure 18. (a) Image of water droplet sitting on the Lotus leaf; (b) Schematic and wetting on nanostructured hierarchical structure. Image reprint from Ref [1]. Directional adhesion on superhydrophobic butterfly wings: (c) The droplet easily rolls along the RO direction when the wing is tilted toward downwards by $9^{\circ}$; (d) The droplet is firmly pinned on the wing that is tilted upward, even when fully upright. Image reprint from Ref [7]; (e) Shape of a water droplet on the petal's surface, indicating its superhydrophobicity with a contact angle of $152.4^{\circ}$; (f) Shape of water on the petal's surface when it is turned upside down. Image reprint from [5].

slightly downward, the droplets begin to roll off the surface in the RO direction. However, when the wing is gradually tilted upwards, even completely vertical, the droplets are firmly pinned to the surface in the opposite direction (Figure 18(d)). Knowledge of surface wettability is still used here in these two different superhydrophobic states, namely the Cassie-Baxer state (with very low adhesion) and the Wenzel state (with high adhesion). This is the direction-dependent rolling and pinning behavior of the droplets on the one-dimensional level, which is related to the specific surface microstructure on the wing described above [7]. The surface of the red rose has a high adhesion to water, and the water droplets do not drip even if the petals are turned over. When the liquid infiltrates micropapillae, but it forms air bump on the nanofolds, this is a kind of Cassie impregnating wetting state.

In summary, the lotus leaf is by far the best natural super-hydrophobic surface, which has inspired many inventions of superhydrophobic, self-cleaning materials. However the surface like rose petals, with high adhesion at high contact angles, can still be used as an imitative object for practical applications.

\section{Replicate the Properties of Natural Superhydrophobic into Applications}

\subsection{How to Replicate the Properties of Natural Superhydrophobic Surfaces to Design Surfaces}

\subsubsection{Design Strategy}

Although there are many superhydrophobic surfaces and also plenty of technologies to build them, the two main requirements for an excellent superhydrophobic surface never change, which are roughness and low surface energy. For example, 
Mingdong $\mathrm{Yu}$ [8] designed a robust and high-performance superhydrophobic surface, by combining organosilicon functionalized $\mathrm{Al}_{2} \mathrm{O}_{3}\left(\mathrm{SiF}_{-} \mathrm{Al}_{2} \mathrm{O}_{3}\right)$ microparticles and low-cost bakelite powder, in order to make the formed surface become rougher than before, as shown in Figure 19. Based on this work, it is easy to understand the design strategy: Produce rough surfaces with hierarchical structure and then modify the rough surface by adding some materials with low surface energy. Typically, roughness plays a bigger role than the low surface energy. For the sake of developing superhydrophobic surfaces, several technologies such as E-beam lithographic, etching, stretching, spin coating, plasma deposition, nanoimprint and colloidal assembly, etc have been explored. The next section, will focus on these different methods to fabricated superhydrophobic surfaces.

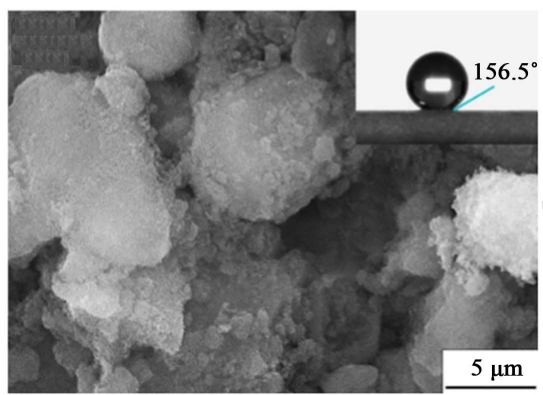

Figure 19. SEM image of $\mathrm{SiF}-\mathrm{Al}_{2} \mathrm{O}_{3}$ microparticles, and the photograph of water droplet on the surface. Image reprint from Ref [8].

\subsubsection{Technologies for Fabricating Superhydrophobic Surfaces}

The techniques which are used to fabricate superhydrophobic surfaces can be categorized into three groups based on the synthesis mechanisms. Top-down means that modify the surfaces via changing the surface structure include several techniques such as lithography, and plasma treatment. Similarly, bottom-up techniques build superhydrophobic surfaces using different methodologies such as self assembly and adsorption. Meanwhile, there are also many techniques that are both Top-down and bottom-up, namely, electrospinning, casting, and nanoimprint. Table 3 lists a variety of superhydrophobic materials and the fabrication methodology.

Table 3. Variety of superhydrophobic materials and the fabrication methodology.

\begin{tabular}{|c|c|c|c|}
\hline Materials & Technique & Contact angle $\left({ }^{\circ}\right)$ & Source \\
\hline PFPS & Chemical polymerization & 152 & $\mathrm{Xu}$ et al. \\
\hline Teflon & Plasma & 168 & Shiu et al. \\
\hline PS nanofiber & Nanoimprint & 156 & Lee et al. \\
\hline FHMA, TIVS & EPD & 105 & Zhong et al. \\
\hline $\mathrm{Si}$ & X-ray lithography Casting & 166 & Furstner et al. \\
\hline $\mathrm{Si}$ & Evaporation & 158 & Sun et al. \\
\hline Carbon nanotubes & LBL & 165 & Lau et al. \\
\hline Silica & LBL & 160 & Zhao et al. \\
\hline
\end{tabular}




\section{1) Lithography}

Lithography is a well-established technique at the heart of a technological process for enabling formation of sophisticated and precise multi-dimensional structures at extraordinary small scales via controlling the morphology of surfaces to create superhydrophobic surfaces. It can allow surfaces patterned with different shapes, and with various parameters. It includes E-beam lithography, X-ray lithography, soft lithography, colloidal lithography, nanoimprint lithography and photo lithography. Even though there are many branches of lithography, their essences are the same. The fundamental principles of this sort of technique produce superhydrophobic surfaces via transferring accurately the devices prefabricated on the template to the surfaces of the wafers and dielectrics according to the required position by the exposure system. For example, Wang [9] successfully created a lotus-leaf-like $\mathrm{SiO}_{2}$ superhydrophobic bamboo surface by using lotus leaves and cross linked PDMS as template and seal for soft lithography. However, there are no clear boundaries between these techniques, in other words, they are multidisciplinary. For instance, the template which is made to obtain the superhydrophobic surfaces gained by soft lithography is also constructed via utilizing the photo lithography.

Photo lithography, the most conventional technique, transfers the geometric patterns prefabricated on the photomask to a slim layer of sensitive photoresist by light, because of general, convenient usage and high efficiency. Soft lithography can be defined as a supplementary expansion of photo lithography. Different from photo lithography, soft lithogarphy can modify an ample rage of elastomeric materials like polymers, gels, organic monolayers. So, the critical elements of soft lithography are elastomeric materials, molds and mask. It is noteworthy that PDMS is the most suitable material for the applications of soft lithography due to its low cost, low toxicity, mechanical flexibility, biocompatibility, as well as durability. And now, the process can also combine with self-assembly and replica molding in order to produce micro- and nanostructures. The electron beam lithography replaces the scattered light that is commonly used in photolithography with focused electron beams to create the surface patterns. X-ray beam lithography is similar to photolithography except for the source of radiation. X-ray radiation is selectively absorbed by the mask and further prepares a pattern. Figure 20 presents a basic common procedure for photolithography.

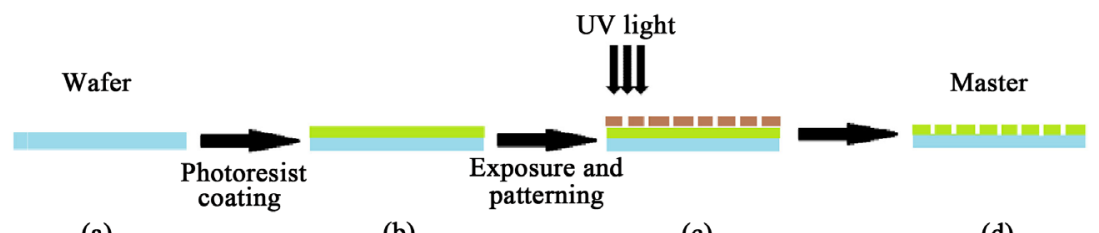

(a)

(b)

(c)

(d)

Figure 20. Schematic of photolithography. (a) A cleaned wafer without any contaminants; (b) Coating the photoresist on the wafer; (c) To place a mask on the photoresist and to expose UV light through the mask; (d) The unexposed part is removed and the surface is got. 
Besides these, nano-imprint lithography is a further development of advanced methods defined as the technique of pressure induced transferring of patterns from a hard mold to a thermoplastic polymer film above its glass transition temperature. Colloidal lithography is advanced method as well which is still under development that utilizes a monolayer of silica colloidal spheres to be masks and prepares the superhydrophobic surfaces by reactive ion etching (RIE).

\section{2) Deposition}

Chemical deposition methods are also a good methodology for producing superhydrophobic surfaces via chemically depositing a thin layer on the substrates. Emblematic chemical deposition methods include electrophoretic deposition, layer-by-layer deposition (LBL), chemical vapor deposition (CVD), and Sol-gel processing. The electrochemical deposition aims to deposit the layers of solid metal or its compound on electrically conductive substrates. The chemical vapor deposition puts the selected substrate in a room that is filled with a gaseous precursor in order to let them have chemical reactions to deposit the craved layer. The LBL deposition is an advanced technique to produce multilayered superhydrophobic surfaces. In this part, we will mainly focus on obtaining superhydrophobic surfaces through these deposition treatments.

\section{a) Layer-by-layer deposition (LBL)}

LBL deposition is a greate method to be used to create superhydrophobic surfaces. Aiming to fabricate slim layer coatings which are controlled over the thickness and chemical properties at molecular level via electrostatic interaction and hydrogen bonding. So the essence of the LBL deposition is changing the charge of substrates and building multilayered films on them. Because of the spontaneous adsorptions of this method, it is unnecessary to provide masks that are widely used in lithography. So the LBL deposition is not only simple but also potentially economical. If we want rough surfaces, adding some nanoparticles into the processes is also a great way, however, the accurate control of surface patterns will be more complex.

Yang and Li's team [10] prepared a fluorine-free superhydrophobic paper based on layer-by-layer assembly. They continuously treated the cellulose fibers with cationic starch (CS) and sodium alginate (SA) to create CS/SA multilayers on their substrate surface. After the LBL deposition, the CS/SA modified cellulose fibers were made into hand sheets via using a semiautomatic sheet former. Superhydrophobicity was increased via immersing the hand sheets into tri-chloromethylsilane (TCMS)-toluene solution for $20 \mathrm{~min}$. The preparation process of superhydrophobic paper is presented in Figure 21. Figure 22 shows the SEM and 3D-AFM morphological images for its hierarchically roughened structures.

In addition, Jindasuwan et al. [11] produced a bio-inspired superhydrophobic surface via imitating the structure of the lotus leaf. They dip coated the superhydrophobic films onto glass by the LBL deposition of polyelectrolyte, and then, modified the surface roughness through depositing $\mathrm{SiO}_{2}$ nanoparticles on 


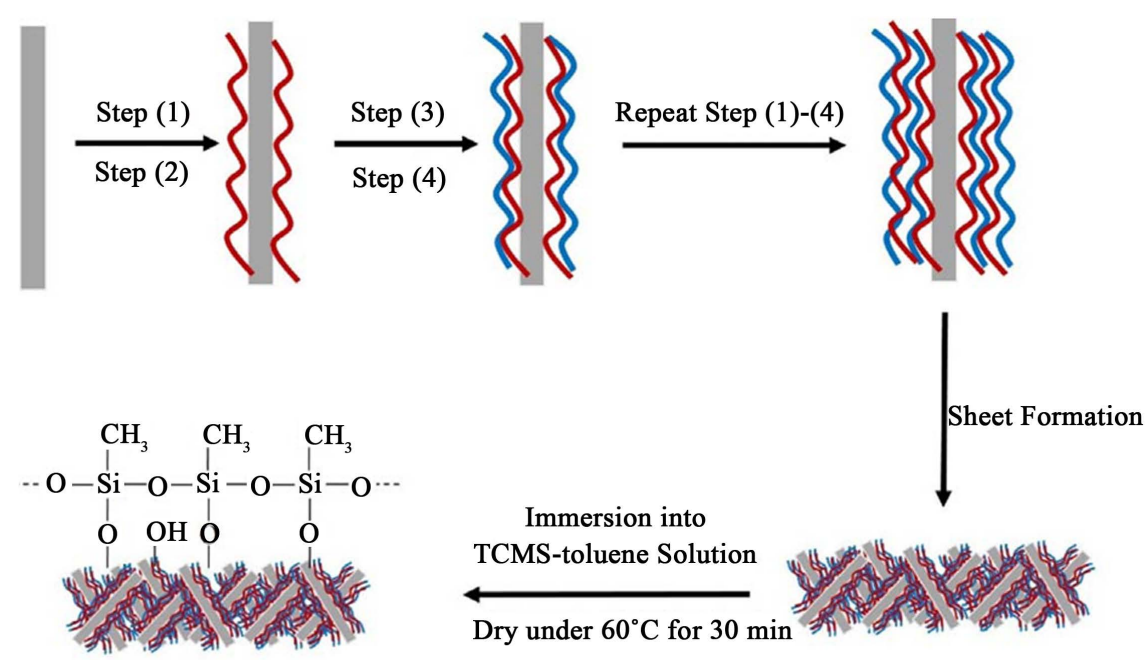

Cellulose Fibers $\sim \mathrm{CS} \sim \mathrm{SA}$

Figure 21. Representation of the preparation process of superhydrophobic paper. Image reprint from Ref [10].

it. Finally, superhydrophobic surface was obtained after applying a semi-fluorinated silane in order to decrease the surface energy.

\section{b) Sol-gel process}

Sol-gel processes have been widely used to fabricate superhydrophobic surfaces according to the Wenzel and Cassie-Baxter model. During the chemical solution deposition, which makes use of sol or solution as a precursor deposited onto the selected substrate in order to create a network, low surface energy materials combined with micro- or nanoparticles can be put into the network as well that will increase the superhydrophobic performance. Considering the transparency, one of the superhydrophobic surfaces' important properties that is typically plagued by its roughness, sol-gel processes have an excellent compatibility with glass, so this method is a perfect way for preparing both superhydrophobic and transparent films on glass surfaces.

Yang et al. [12] recently reported a facile, green and low-cost sol-gel process for the creation of superhydrophobic cotton fabric coated by fluorinated $\mathrm{TiO}_{2}$ sols. In addition, the fluorinated $\mathrm{TiO}_{2}$ sols were got by the prepared $\mathrm{TiO}_{2}$ sols catalyzed them by acetic acid and modified by poly (Hexafluorobutyl methacrylate) with (3-mercaptopropyl)trimethoxysilane (MPTS) as the chain transfer agent. Figure 23 shows the reaction route of fluorinated $\mathrm{TiO}_{2}$ sol preparation and coating procedure. Similarly, Shirtcliffe et al. [13] prepared porous sol-gel foams from varying proportions of phenyl triethoxysilane and TEOS whose performance switched between superhydrophobicity and superhydrophilicity by changing different surrounding temperatures.

Conventional sol-gel process employing specific organo-silane and polyhedral oligomeric silsesquioxane as precursors have also been considered for preparing superhydrophobic coatings. However, a light-driven route for superhydrophobic 


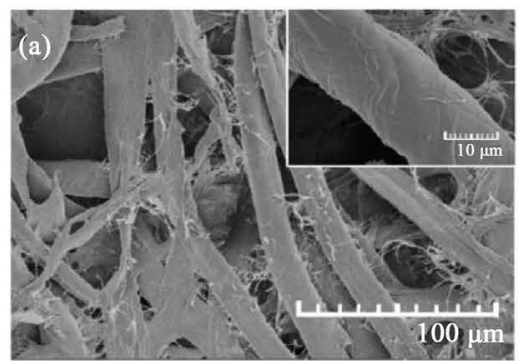

(a')
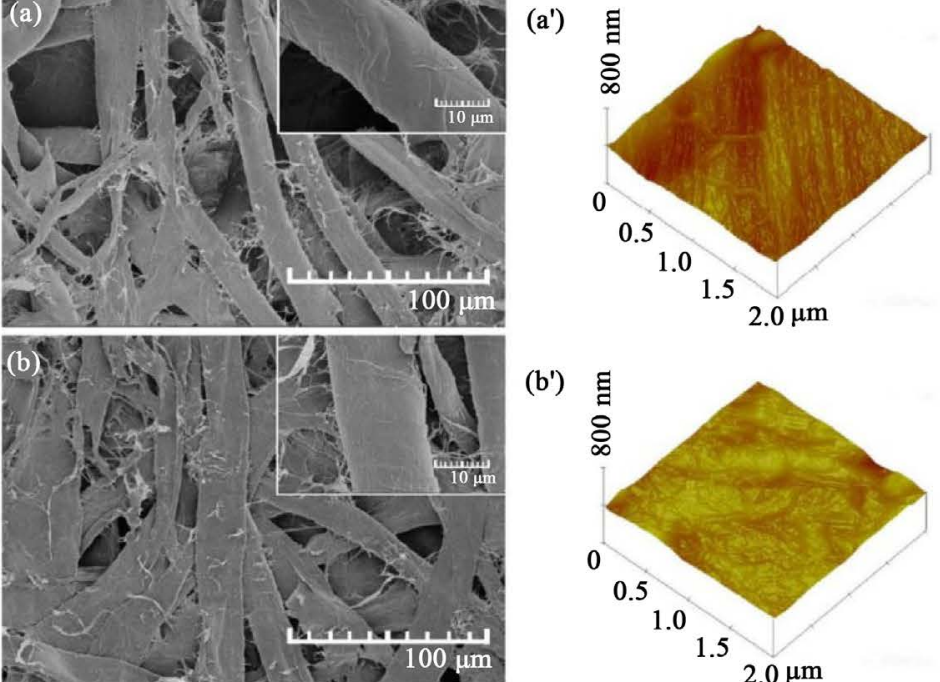

(b')
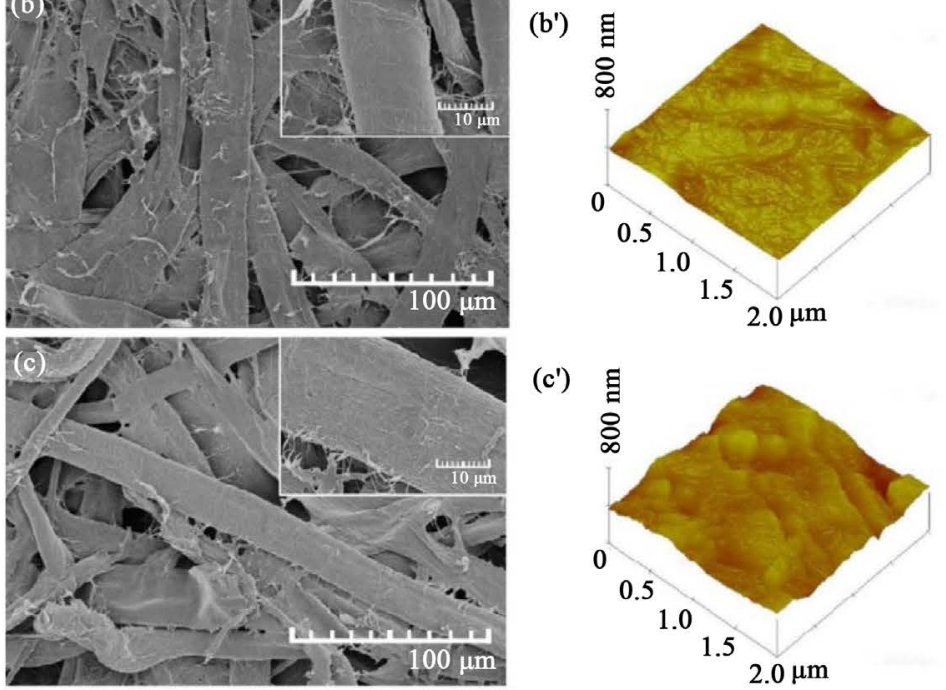

(c')
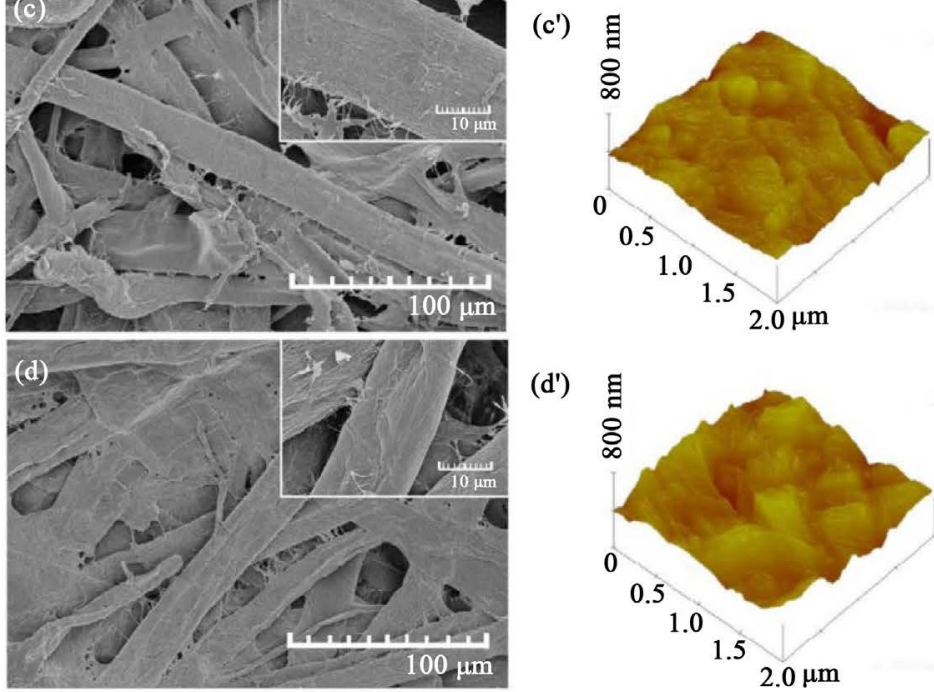

(d')
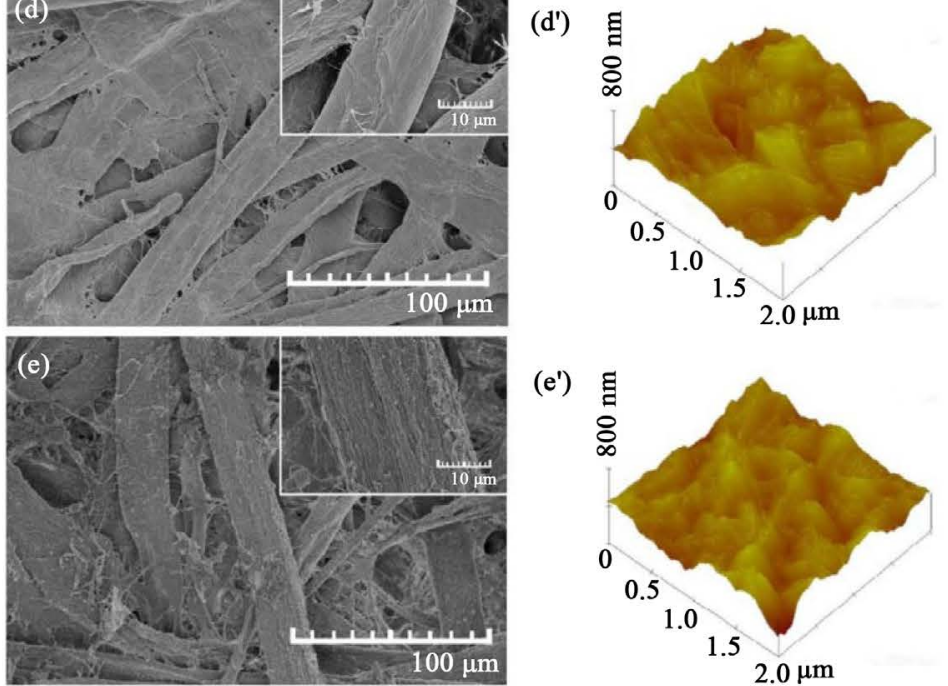

(e')

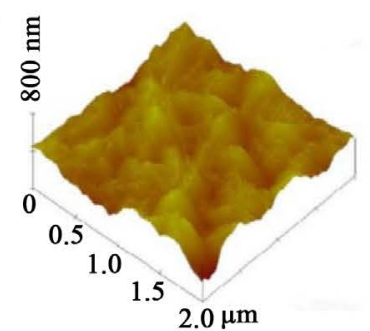

\begin{tabular}{cc}
\hline Samples & RMS roughness \\
\hline Control & 29.02 \\
(CS/SA) & 18.31 \\
(CS/SA) & 35.98 \\
$(\mathrm{CS} / \mathrm{SA})_{5}$ & 53.67 \\
TCMS-(CS/SA) & 68.52 \\
\hline \multicolumn{2}{c}{$(\mathrm{f})$}
\end{tabular}

Figure 22. SEM and 3D-AFM morphological images for different samples. (a) (a') pristine paper. (b) (b')-(d) (d') paper modified with a $(\mathrm{CS} / \mathrm{SA})_{1},(\mathrm{CS} / \mathrm{SA})_{3}$ and $(\mathrm{CS} / \mathrm{SA})_{5}$ multilayer. (e) (e') paper modified with a TCMS-treated $(\mathrm{CS} / \mathrm{SA})_{5}$ multilayer. Image reprint from Ref [10]. 


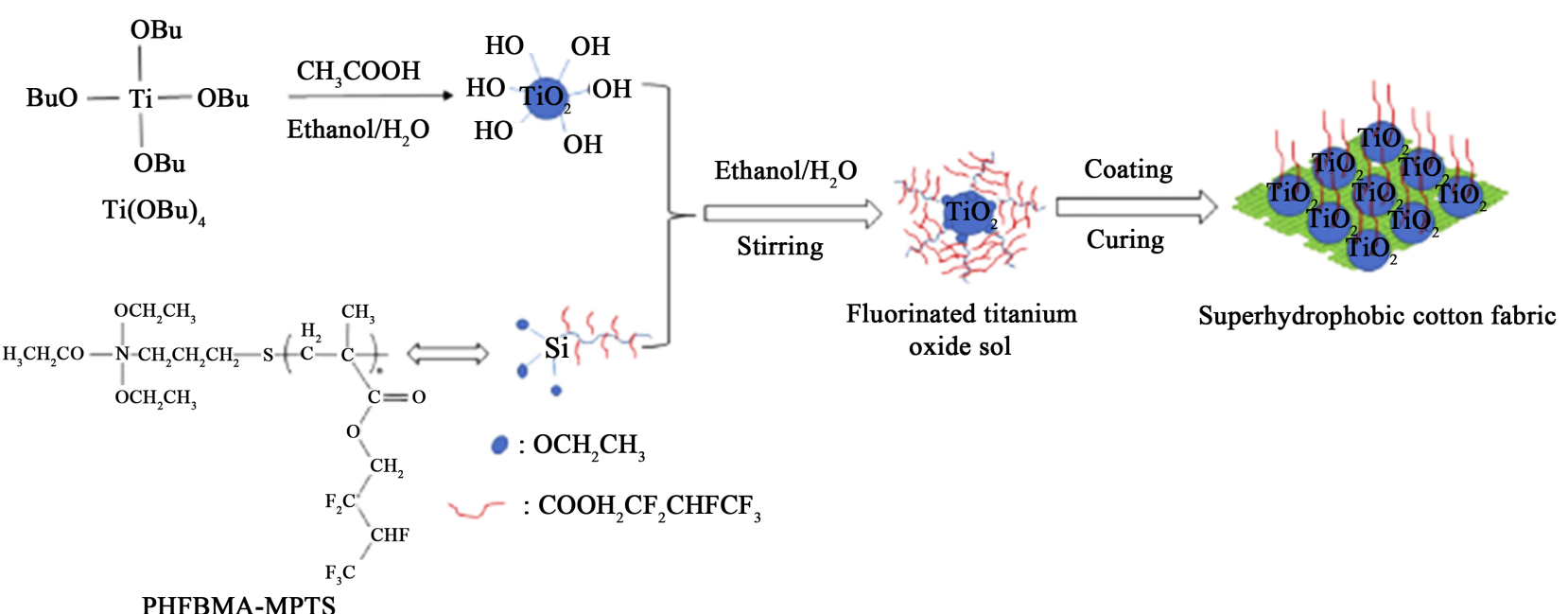

Figure 23. The reaction route of fluorinated $\mathrm{TiO}_{2}$ sol preparation and coating procedure. Image reprint from Ref [12].

coatings involved with volatile organic solvents either to ensure deposition or to induce roughness is an advanced development of a radiation-mediated sol-gel process which is recognized as both the eco-efficient and the effective technology. For instance, $\mathrm{Ni}$ et al. [14] reported a superhydrophobic sol-gel coating produced in a one-step from a single precursor by the light-induced condensation of a $\alpha, \omega$-bis-silylated alkane which can construct a superhydrophobic hierarchical structure driven by the self-assembly of the organo-silica network (Figure 24).

\section{c) Aqueous electrophoretic deposition}

Aqueous electrophoretic deposition is a potential new strategy for the preparation of superhydrophobic surfaces on conductive substrates. This deposition refers to the deposition of colloidal particles onto substrate surfaces by direct current electric field in a stable suspension. Moreover, it also has a number of advantages, in comparison with existing techniques, including its environmental-friendly and cost-effective features, its potential for the production of coatings for conductive substrates with complex shapes, and safety. Although the use of water as a solvent can weaken the adhesion between a coating and substrate because of de-wetting effects during drying, special water-soluble precursors can solve this issue. As an example, Zhong et al. [15] prepared a kind of robust anti-smudge coating via electrophoretic deposition. This team designed a novel and practical water-soluble polyacrylate (WPA) as the precursor for the aqueous electrophoretic deposition that includes poly (methyl methacrylate) (PMMA). Ideal components were selected for enhancing the coating transparency, the 2-(Dimethylamino)-ethyl methylacrylate (DMAEMA) for providing water-solubility, 2-hydroxypropyl methacrylate (HPMA) for preparing strongly the crosslinked coating matrix. They also chose 2-(perfluorohexyl)ethyl methacrylate (FHMA) and tri (isopropoxy)vinylsilane (TIVS) which have low surface tensions and can enable the coating to adhere tightly with conductive substrates. Figure 25 shows more details about this work. 

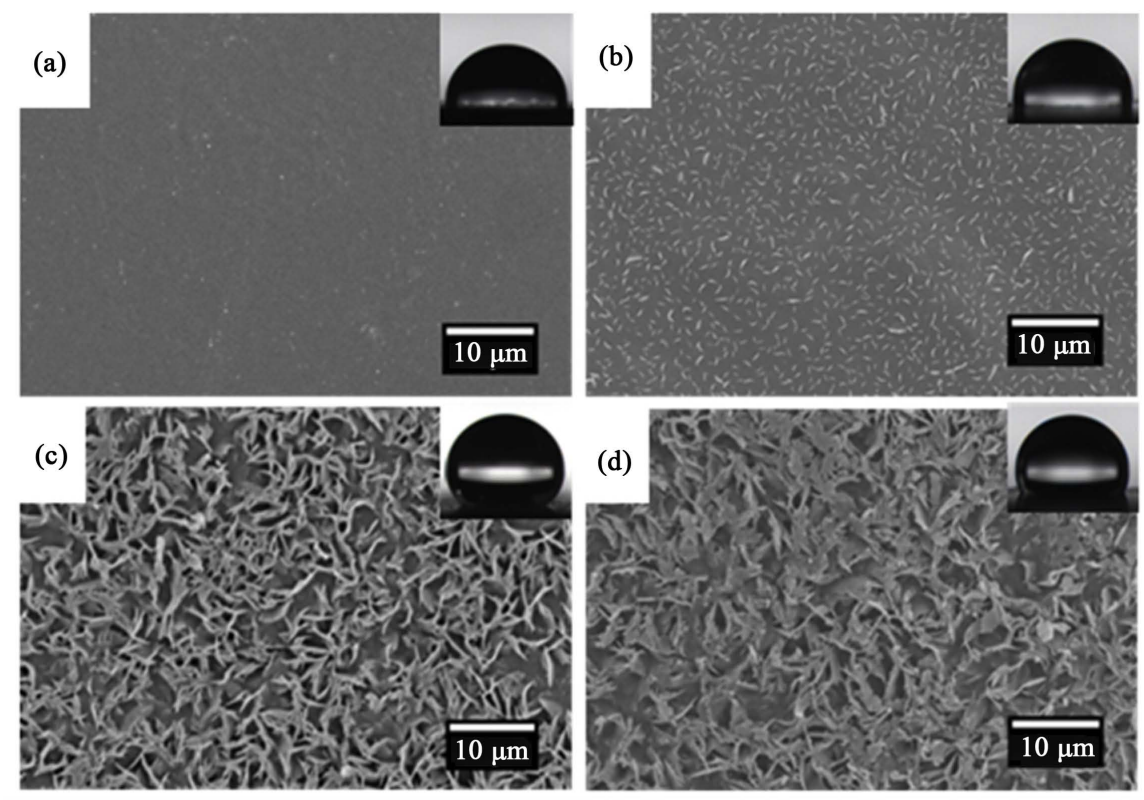

Figure 24. SEM of $\mathrm{BC}_{6} \mathrm{SQ}$ prepared at different irradiances: (a) 20; (b) 2; (c) 0.5 ; (d) 0.1 $\left(\mathrm{mW} \cdot \mathrm{cm}^{-2}, 30 \mathrm{~min}\right.$ irradiation). Image reprint from Ref [14].

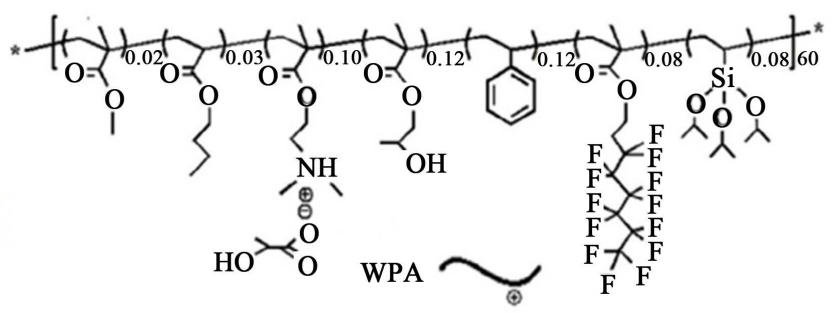

(a)

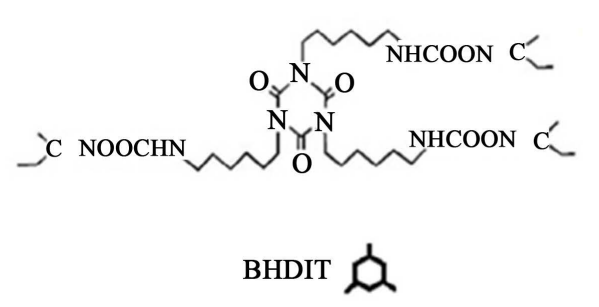

(b)

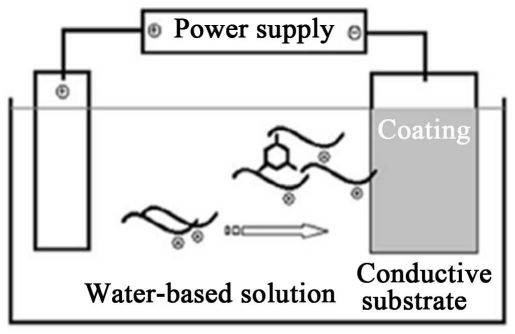

(c)

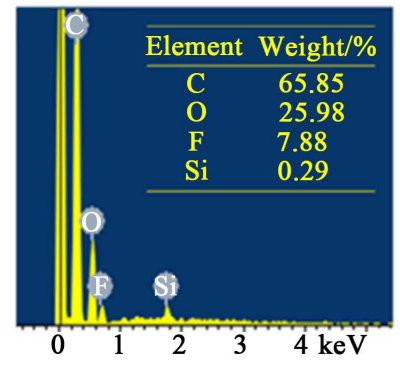

(d)

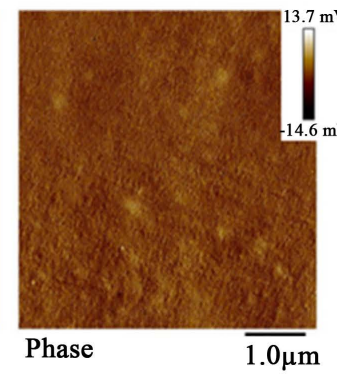

(e)

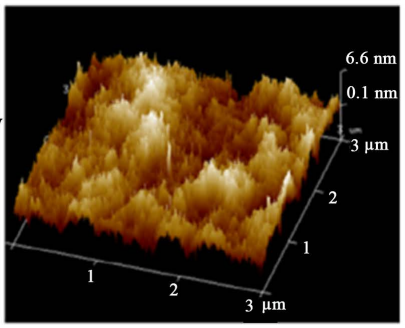

(f)

Figure 25. (a) The structure of WPA; (b) and BHDIT; (c) Illustration of the EPD protocol; (d) The elementary analysis of the coating by SEM-EDS; (e) AFM image and (f) a 3D topography image of the coating. Image reprint from Ref [15].

\subsubsection{Hydrophobic Materials}

Modifying a rough surface with materials of low surface energy is also a common technique to make superhydrophobic surfaces. Hydrophobic materials/Low surface energy materials are as important as roughness in fabricating superhydrophobic surfaces. The common hydrophobic materials are fluorocarbons, organic materials, inorganic materials and silicones. We will briefly introduce these materials as following. 


\section{1) Silicones}

Polydimethylsiloxane (PDMS) is a well-known material with low surface energy and elasticity. Given these critical elements, PDMS has become one of the most popular materials for making superhydrophobic surfaces through various methods. Jia et al. [16] successfully obtained a homogeneous molecular-level dispersion of rigid vinyl-polyhedral oligomeric silsesquioxane (V-POSS) into flexible PDMS matrix named poly (dimethylsiloxane)-polyhedral oligomeric silsesquioxane (PDMS-POSS) coating with cauliflower-shaped beads via electrospraying with polyvinylidene fluoride (PVDF). The water contact angle (WCA) for the treated surface was as high as $166^{\circ}$, evidence for the superhydrophobicity of PDMS-POSS surface (Figure 26). Similarly, Liu et al. [17] created biomimetic PDMS films with superhydrophobicity, self-healing and drag reduction via the replication of the micron-sized grooved structure of shark skin and the coating with poly (2-perfluorooctylethyl methacrylate) (PFMA) brush and $\mathrm{SiO}_{2}$ particles. Such a surface showed a superhydrophobicity with WCA exceed $160^{\circ}$ and sliding angle lower than $2^{\circ}$ (Figure 26).

Using a block copolymer like poly (styrene-b-dimethylsilox-ane) is also a significant way to utilize the low surface energy of PDMS. Zhang et al. [18] provided a strategy to prepare PDMS/P (VDF-TrFE)/PS surface exhibiting high permittivity and superhydrophobic properties. They chose PDMS and ferroelectric poly (vinylidene fluoride-co-trifluoroethylene) (P (VDF-TrFE)) as the polymer matrix because of the high permittivity and hydrophobicity. To further enhance these properties, they synthesized PS microspheres via emulsion polymerization and embedded them into PDMS/P (VDF-TrFE). As a result, the surface presented a WCA of $125^{\circ}$.

\section{2) Fluorocarbons}

As well-known polymers with low surface energies, fluorinated polymers are ideal materials for producing superhydrophobic surfaces directly. For example, Sarkar et al. [19] treated aluminum surfaces with dilute hydrochloric acid via chemically etching, and, in order to enhance the contact angle, coated the

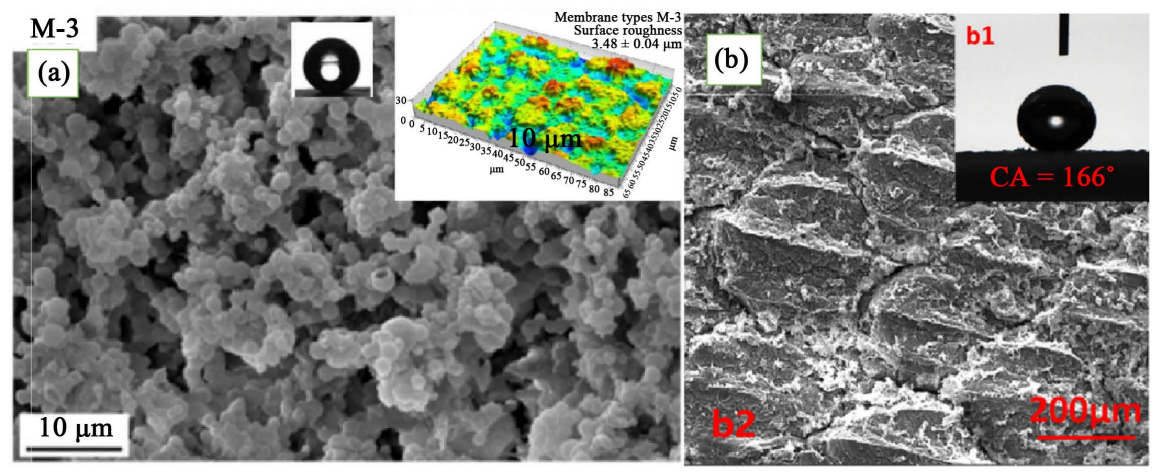

Figure 26. (a) SEM image of the top surface for the membrane, topology and surface roughness of the membrane, and the WCA. Image reprint from Ref [16]; (b) The WCA photograph and SEM image of PDMS films are shown in b1, b2 respectively. Image reprint from Ref [17]. 
surface with ultrathin rf-sputtered Teflon film. Thus, a water contact angle as high as $164^{\circ}$ with a contact angle hysteresis as low as $2.5^{\circ}$ has been presented. The existence of $-\mathrm{CF}_{3}$ and $-\mathrm{CF}_{2}$ groups in the ultrathin $R_{f}$ sputtered Teflon films impressively decreases overall substrate the surface energy (Figure 28). Polytetrafluoroethylene (PTEE) is also both stable and low surface energy material. $\mathrm{Zhu}$ et al. [20] used coaxial electrospinning to fabricate a hierarchical $\mathrm{SiO}_{2} / \mathrm{PTEE}$ nanofiber membrane and viewed $\mathrm{SiO}_{2}$ nanoparticle dispersion as the shell spinning solution and PTEE as the core spinning solution via in-situ surface grafting of $1 \mathrm{H}, 1 \mathrm{H}, 2 \mathrm{H}, 2 \mathrm{H}$-perfluorooctyltriethoxysilane. Furthermore, the treated membrane displayed excellent durable superhydrophobic properties with a water contact angle of $173^{\circ}$ (Figure 27). In addition, $\mathrm{Xu}$ et al. [21] created a double-roughened perfluorooctanesulfonate (PFOS) mixed with polypyrrole (PPy) through the synergy gained from electropolymerization and chemical polymerization. This surface can also change its surface property from superhydrophobicity to superhydrophilicity in response to different applied electrochemical potential.

\section{3) Organic materials}

Besides silicones and fluorocarbon, organic materials that contain various paraffins, a kind of hydrophobic material, existed in nature creatures such as lotus, butterfly, bird etc. widely. So using these materials to prepare superhydrophobic surfaces will not only be cheap but also green. Cheng et al. [22] designed a straightforward, safe and inexpensive strategy using high density polyethylene (HDPE)SS coatings based on polymer drop-casting to produce different superhydrophobic

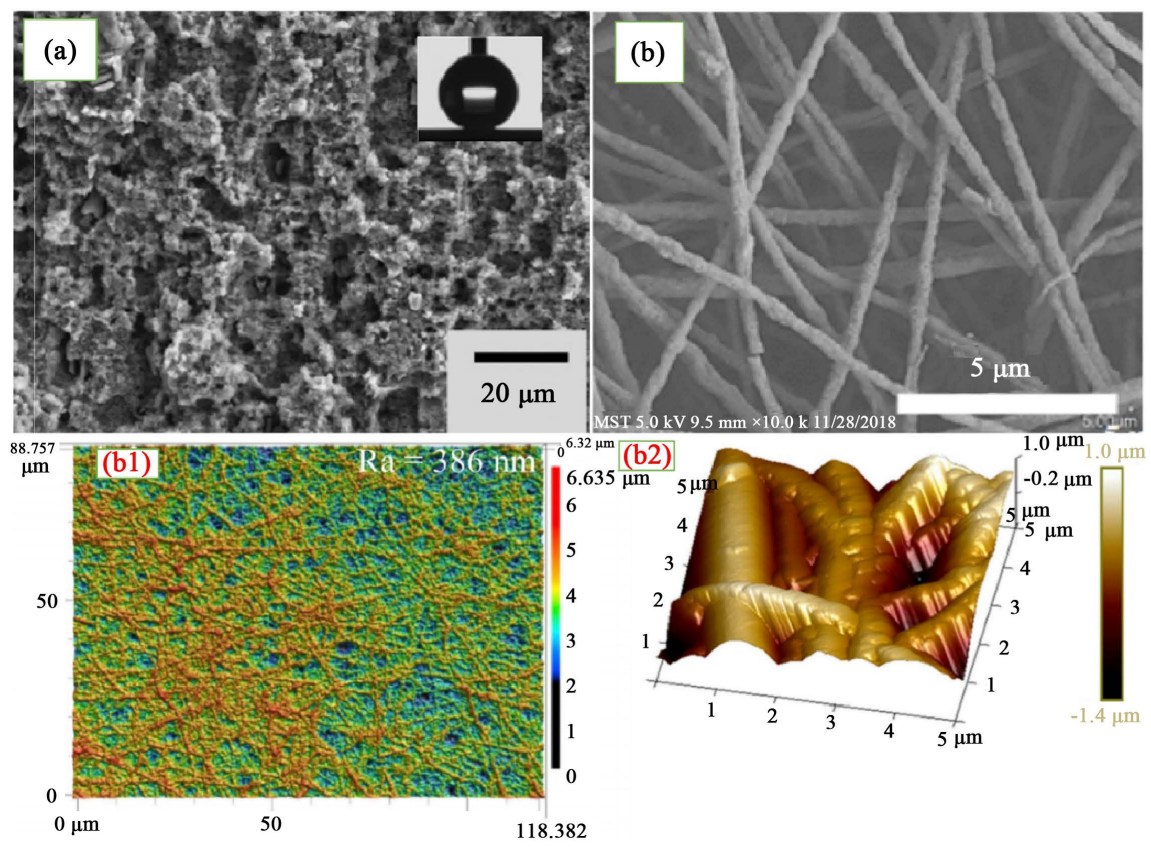

Figure 27. (a) FESEM images of ultrathin fr-sputtered Teflon coated aluminum substrates etched with 14.8 wt\% HCL and the WCA. Image reprint from Ref [19]; (b) SEM image, and 3D AFM image and surface topography image are shown in (b1), (b2), all of the $\mathrm{SiO}_{2} /$ PTEE. Image reprint from Ref [20]. 
surface with several substrates include glass, copper mesh, and polyethylene (PU) sponge as shown in Figure 28. Each of these substrates displayed a superhydrophobic behavior even with a water contact angle as higher as $150^{\circ}$ even if glass is hydrophilic before. Moreover, the treated glass, copper mesh, and PU sponge also showed fine ability for corrosion resistance and stability. Liang et al. [23] created alkenyl-functionalized $\mathrm{SiO}_{2}$ particles (AFSPs) and grafted polystyrene (PS) onto them to synthesize $\mathrm{SiO}_{2}$-g-PS particles through emulsion polymerization of styrene. Then, the AFSPs were allowed to grafted and combined with fluoroalkyl silane to prepare superhydrophobic and oleophobic coatings. Similarly, Zhang et al. [24] exploited a novel dual-templating method based on the High Internal Phase Emulsions (HIPEs) processing and the introduction of organic template species such as calcium stearate which are added into the HIPEs to increase roughness and give the PolyHIPEs with hydrophobicity in order to create alow density polystyrene sponge that exhibited superhydrophobicity

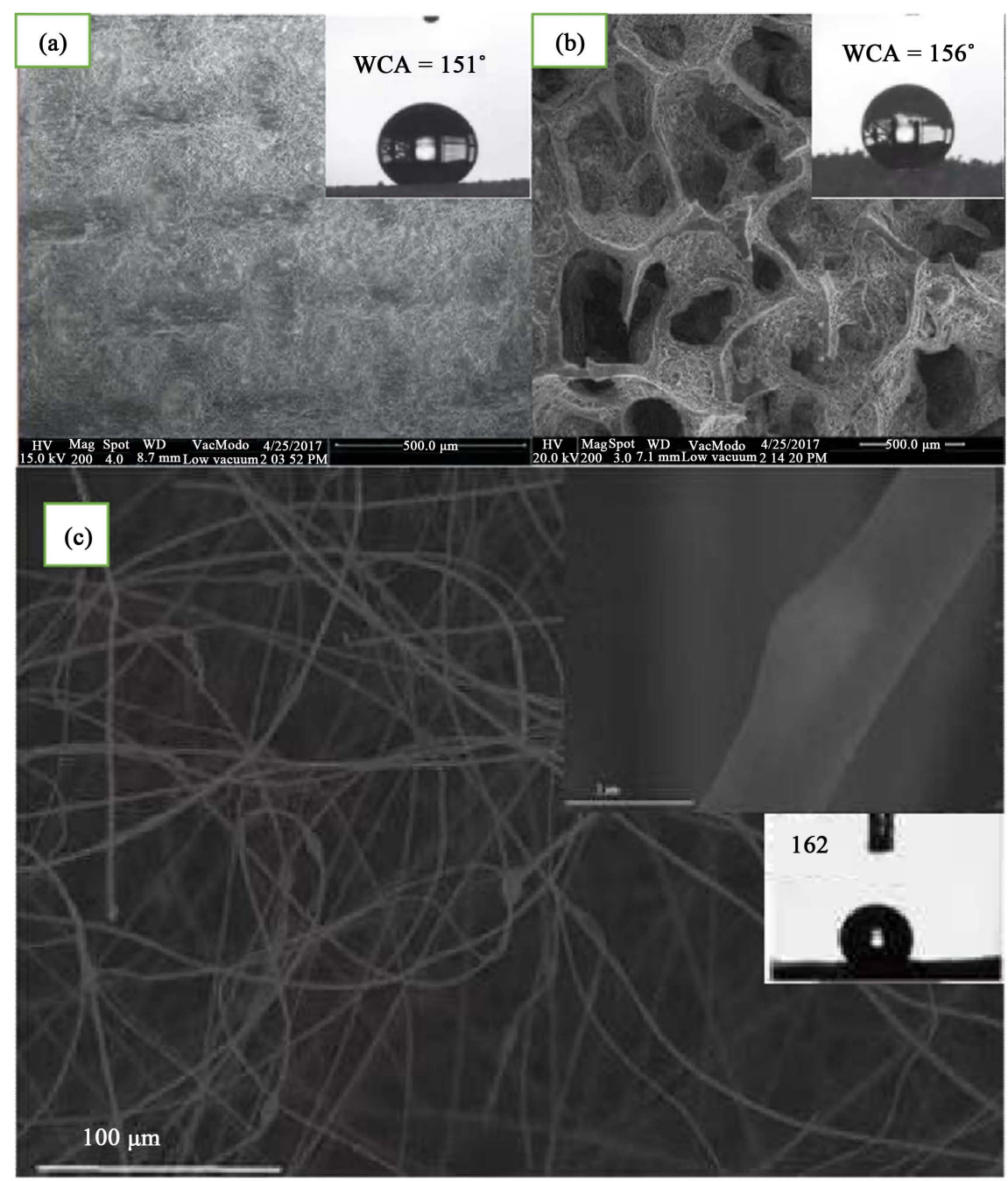

Figure 28. (a) SEM image and WCA of copper mesh; (b) SEM image and WCA of PU sponge; (c) FESEM image of $\mathrm{Fe}_{3} \mathrm{O}_{4}$ intercalated PS nanofiber mats with $10 \mathrm{wt} \%$ and its WCA. Image reprint from Ref [22]. 
and compressibility. Considering the poor solubility of calcium stearate in ethanol, it is easy for calcium to remain in the PolyHIPEs and further forming abundant small bulges which effectively enhance the roughness of the surfaces with WCA higher than $150^{\circ}$. Polystyrene can also be associated with other things to fabricate superhydrophobic surfaces. For example, Sara M. Moatmed et al. [25] designed a highly efficient and reusable superhydrophobic polystyrene/ $/ \mathrm{Fe}_{3} \mathrm{O}_{4}$ nanofiber membrane by using electrospinning with WCT $162^{\circ}$.

\section{4) Inorganic materials}

Some particular inorganic materials are appropriate choices for decorating superhydrophobic surfaces as well, which means low environmental contamination and material cost. Usually, the deposition is a good way for these materials in order to enhance the surfaces roughness. For example, Cheng et al. [26] reported a $\mathrm{NiO} / \mathrm{ZnO}$ superhydrophobic surface with a WCA of $153^{\circ}$ which has the corrosion resistance, low hysteresis and anti-abrasion character via chemical deposition using zinc substrate as the starting materials, and then thermal annealing at $170^{\circ} \mathrm{C}$ for $90 \mathrm{~min}$ in air subsequently. This strategy does not need any additional modification with organic films, and the superhydrophobic surface is created only through deposition to obtain surface morphology with high roughness, and thermal annealing to change the composition (Figure 29). In addition, He et al. [27] obtained a durable superhydrophobic $\mathrm{Zn} / \mathrm{ZnO} / \mathrm{TiO}_{2}$ surfaces with dendritic structures on $\mathrm{Ti}_{6} \mathrm{Al}_{4} \mathrm{~V}$ substrate through chemical etching, electro deposition at $-1.5 \mathrm{~V}$ for $10 \mathrm{~min}$, and annealing process at $190^{\circ} \mathrm{C}$ for $60 \mathrm{~min}$. $\mathrm{Al}-$ though there is no organic modification, the treated surface also exhibited perfect superhydrophobicity; the WCA was $160^{\circ}$ and the sliding angle was less than $1^{\circ}$. Moreover, reversible superhydrophobicity-superhydrophilicity switching was showed by the $\mathrm{Zn} / \mathrm{ZnO} / \mathrm{TiO}_{2}$ surface as well via UV irradiation and heating treatment.

\subsection{Design and Application of Superhydrophobic Surface in Several Aspects}

Heretofore the bio-inspired non-wetting surfaces have drawn grand and wide attention around the world. Different kinds of superhydrophobic surfaces which possess different chemical compositions and structures have different applications. Recently, the superhydrophobic surfaces are not only applicated in the medical treatment, but also in the transportation, things associate with our daily lives closely. Considering that, it is obvious that the superhydrophobic surfaces are more and more necessary, its status becomes more and more critical. In this part, we will mainly talk about the designs and applications of superhydrophobic surfaces in diversity fields.

\subsubsection{Application in the Transportation}

\section{1) Anti-icing}

Ice is a beautiful crystal with six branches. But, in transportation, ice is a really 

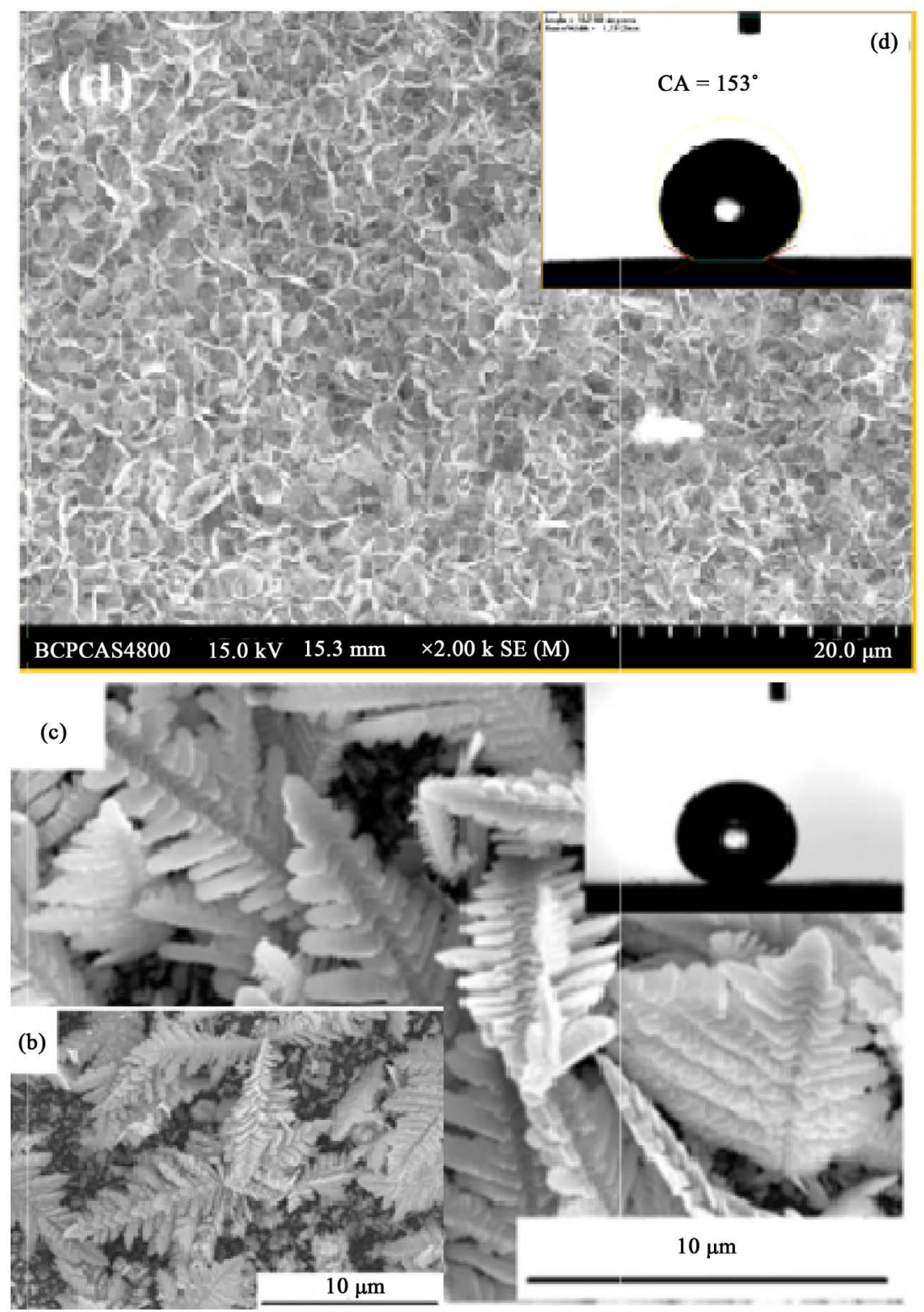

Figure 29. (a) SEM image of the $\mathrm{NiO} / \mathrm{ZnO}$ superhydrophobic surface and the WCA. Image reprint from Ref [26]; (b) SEM images of the $\mathrm{Zn} / \mathrm{ZnO} / \mathrm{TiO}_{2}$ surface and the sample after UV irradiation and then heating and the WCA. Image reprint from Ref [27].

hazardous thing which can cause several safety accidents leading to both economic losses and the casualties. Thus, to develop ice resistant or ice-phobic coatings for automobiles, naval vehicles, aircrafts, wind turbines, satellite dishes and rockets is a critical need. To evaluate the anti-icing performance, there are two ways: 1) How to remove the ice from the surfaces easily. 2) How to delay the nucleation time before the water droplets crystallize. Unfortunately, common superhydrophobic surfaces with low surface energy and high roughness texture will enhance ice crystallization under high humidity conditions. Although, contrast to this point, some experts think that perfect hydrophobicity has a great potential to prevent ice formation because the water can slide down quickly be- 
fore it freezes. However, in fact, the roughness did not help with the anti-icing performance, or even made it worse, as it let the water from air to be condensed inside the grooves and produce nucleating sites in which ice can be assembled. Meanwhile, Bharathidasan et al. [28] suggested that ice adhesion is lower on smooth hydrophobic silicone coatings that on the rough superhydrophobic surfaces. Although the roughness did not work, the extreme low surface energy can prevent the creation of ice. Srinivas et al. [29] reported that because of the inhibition of frost formation within the nanofeatures and the stabilization of vapor pockets, the ice adhesion which obviously increased on micro-surfaces would have a 15-fold decrease on nano-surfaces. Li et al. [30] designed a non-toxic, low-cost method to prepare a hierarchical superhydrophobic surface on aluminum alloy through the combination of laser-fabrication and chemical etching, and then chemical vapor deposition of PDMS to yield a WCA of $155^{\circ}$ which also exhibited the low ice adhesion strength. Another strategy to create a pliable anti-icing surfaces is through the low pressure plasma polymerized hexamethyldisiloxane coating was as suggested by Mobarakeh et al. [31] The coating presented both low ice adhesion strength and high durability. Considering about all these works, we can get the conclusion that despite the superhydrophobic surfaces have some disadvantages for icing resistance, it is also viable to design special superhydrophobic surfaces in anti-icing applications via particular treatments.

\section{2) Self-cleaning}

The existence of contamination often seriously affects the efficiency and safety of transportation. Self-cleaning is an important and interesting property of superhydrophobic surfaces which contain the rough surfaces, which is crucial for self-cleaning through compositing with photocatalyst and the low surface energy. Recently, a superhydrophobic $\mathrm{Cu}-\mathrm{Ni}$ coating was produced by Liu et al. [32] through the combination of electro-deposition and myristic acid modification. Through the experiment, the combination of hierarchical structure and low surface energy of the treated surface results that the contaminants can be easily removed showing the excellent self-cleaning. Zhang et al. [33] reported a facile fabrication of nonfluorinated superhydrophobic aluminum surfaces through one step electro-deposition. In the following experiment, Zhang tested the self-cleaning and anti corrosion. They spread out graphite powder as contaminants over the treated surface and added water to it which easily slid off the surface and took the contaminants away. It results that this surface had a fine contaminant repellent performance. $\mathrm{Xu}$ et al. [34] have developed a simple hydrothermal method for in-situ fabricate $\mathrm{CuO}$ (micro/nano-structures) spheres on polyimide. Interestingly, this surface can not only resist corrosion and harsh condition, but also have a super self cleaning character for both organic and inorganic contaminants.

\subsubsection{Application in the Medical Treatment \\ 1) Control of Protein adsorption}


Proteins are one of the most critical biomolecules in our body and play very important roles in our lives where have many functions such as protein transport, enzyme regulates, antibody. However, when we use medical devices to treat ourselves, the protein would like to adsorb on the material surfaces, while it can lead to various deleterious symptoms like hemolysis. So it is very urgent to control the protein adhesion. One way to achieve this goal is to make the surfaces have extremely high roughness and low surface energy. Nowadays, there are some scholars suggest that the rougher the surfaces on the micron scale, the more the number of proteins adhering on it. However, For instance, Tsougeni et al. [35] designed rough surfaces which were hydrophobized by means of a $\mathrm{C}_{4} \mathrm{~F}_{8}$ plasma deposition step via a stencil mask producing superhydrophobic surfaces and present an extraordinary ability in decreasing protein adsorption. According to this result, it is apparent that superhydrophobic surfaces with hierarchical structures and nano-scale roughness can have good performance in protein adsorption.

\section{2) Control of bacteria adhesion}

Bacteria, small organisms, are closely related to our lives. However, not all bacteria's are beneficial for our health. For example, pathogenic bacteria usually cause several serious diseases. To face that, humans have developed many antibiotics and medicines, but the overuse of drugs can cause bacteria to be drug resistant. Thus, a new strategy to arrest them becomes more and more emergent. Zhang et al. [36] reported a simple etching-textured superhydrophobic 5083 aluminum alloys (AA5083) with micro-nano hierarchical structure and $1 \mathrm{H}, 1 \mathrm{H}$, 2H, 2H-Perfluorodecyltriethoxysilane (PFDTES) in order to suppress bacteria adhesion. They immersed the prepared surface in the bacteria culture medium and found that the surface was covered by nearly no bacteria, while the comparison was covered with coverage of $11.99 \%$ (Figure 30). Moreover, Shen et al.

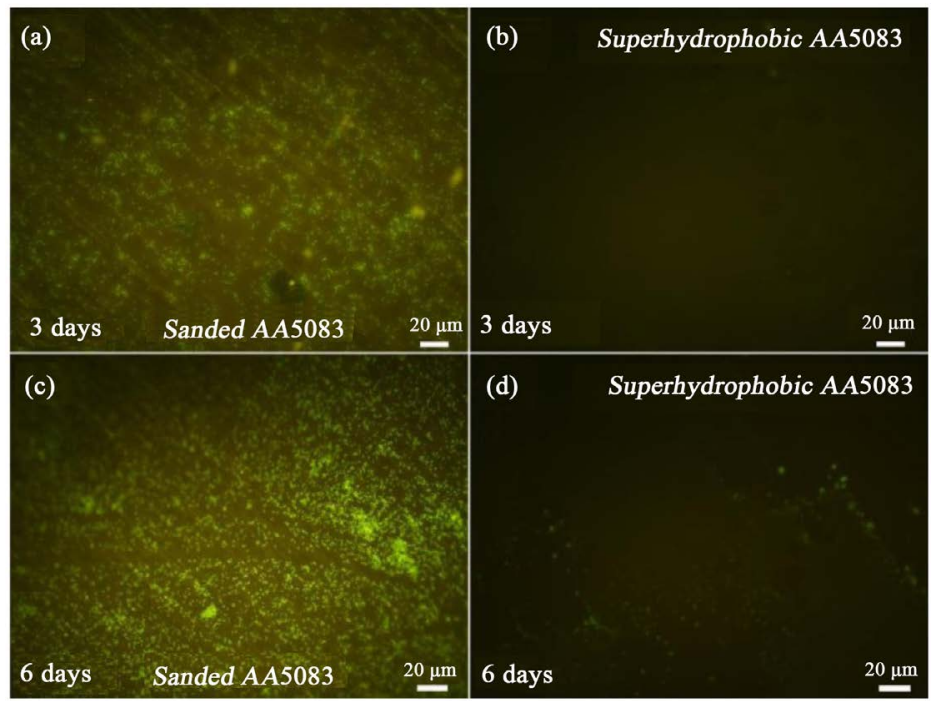

Figure 30. Fluorescent micrographs of the raw AA5083 (a) (c) and the treated AA5083; (b) (d) after 3 days and 6 days bacteria culture medium immersion. Image reprint from Ref [36]. 
[37] posed a novel way to reduce bacterial load that is using a superhydrophobic coating to minimize the number of bacteria adhering on a surface and killing them through antibacterial agent. All the works mean that the superhydrophobic surfaces are emerging materials and tools for people to the fight against diseases.

\section{The Limitation of the Current Generation of Superhydrophobic Surfaces and Prospective}

\subsection{The Limitation of the Current Generation of Generation of Superhydrophobic Surfaces}

It is certain that superhydrophobicity is a recently appeared concept. But the result is, this concept has caused great attention and produced countless positive effects. According to the basic knowledge of this discipline, such as the Wenzel, Cassie-Baxter models and the wetting transitions between these two models which all have been mentioned before, scientists have created numerous theoretical models for producing the materials which have the ideal properties. The difficulties of exploring procedures are not invisible to us as the interconnected nature of this concept. Actually, making sense of this discipline is high demanding on account of its connections to different kinds of fields, including physics, chemistry, material science, sometimes even biology. As to the limitation of the current researching devices, researchers need to address many problems without the most stable and powerful theoretical explanations. Experimental data are insufficient. Based on the situation, nature is the most significant consultant. Bionics has participated in the researching field with the strongest influences. Scientists have produced extensive materials based on the advantages that some natural creatures' composition has. As a result, many of the products have commercialized and caused great convenience to people's life. However, there are still lots of limitations to the current researching. As mentioned before, currently the satisfactory experimental devices have not come out yet [2]. If we do not have the experimental data, we could not offer the best explanation for some natural materials or even corroborate the practicality of the theoretical models that scientists have provided. The needed exploration will be a long journey according to the difficulties we have faced today. For the second thing, Young equation, Wenzel equation, and Cassie-Baxter equation are the fundamental theories for this concept. But these theories do not satisfy all the situations. When the droplets wet the superhydrophobic surface, its state is dependent on the properties which the surfaces have and the wetting process. The transition between these states may appear because of the changes in energy balances. Apparently, an equation or a theoretical model which fits all the situations has not appeared yet. Besides, the proper control of the roughness that caused the transitions between these states has become the main challenge to provide a stable hierarchical surface [38].

\subsection{Prospective}

According to the recent development of superhydrophobic surfaces, we could 
have a positive view toward the further growth of our knowledge about the concept while using them to create the materials that meet our demands. With further works into the field of superhydrophobicity, the most basic problem that we need to get over is precisely controlling the roughness of the surface in both small and large scales. On account of the precise control needed to base on our understandings of the wetting procedures, we need to make out the essences of wetting procedures. Especially the transitions between states on the superhydrophobic surfaces, we need to apply the fundamental theoretical models which could fit all conditions [2]. Secondly, the contemporary applications of superhydrophobic surfaces are mostly in self-cleaning materials. It is demanded that we have to make a deeper understanding the rationale behind these materials which could help us to mimic and develop them to make other applications, such as exterior paintings, high-power lines, and solar penal. Apply them into these fields could help us convert the energy while keeping the smallest loss and temporarily conserve the energy [39].

Besides, these materials could be used on drug packaging. If we could reduce the wet area available for bacteria absorption, their growth could be limited for even several days so that antibacterial agents could be released over time and have the strongest and the most ideal effect. Similarly, superhydrophobic materials could be used in preventing of blood coagulation. Superhydrophobic surfaces perform reduced platelet attachment and coagulation with increased contact angle. Therefore, blood compatibility is observed only in brief periods of time. Superhydrophobic materials can also be used for the tunable delivery of drugs. The ability to control wetting is used to slow dissolution of drug entrapped in superhydrophobic meshes, coatings. Drug release is demonstrated for weeks under rigorous in vitro conditions, and efficacy is observed in an in vivo model of local cancer recurrence.

There are several additional applications of this new technique, such as 1) surface materials which can keep Cassie-Baxter state in protein solutions for months which are the improvements of the former technology which could only maintain them in the Cassie-Baxter state for only several weeks; 2) as contemporary materials which could deliver proteins are basically focusing on small molecule, we could search for more diverse materials based on our new findings; 3) exploring materials which possess the qualities of stimuli-responsive and reversibly wetting superhydrophobic or superhydrophilic materials [40].

More potential applications for biomaterial product, such as 1) materials which could reduce bacterial adhesion when in contact with blood or bodily fluids using the superhydrophobic coating; 2) discover materials for disposable microfluidic diagnostic devices where the superhydrophobic coating supports droplets; 3)find coated medical devices for drug delivery in oncology [40].

\section{Acknowledgements}

The authors wish to thanks prof. Anish Tuteja who excels in studying superhydrophobic surfaces, he has been helped us to modify and improve in the process 
of writing the paper. This work was supported in part by a grant from CIS Academic Office.

\section{Conflicts of Interest}

The authors declare no conflicts of interest regarding the publication of this paper.

\section{References}

[1] Bhushan, B. and Jung, Y.C. (2011) Natural and Biomimetic Artificial Surfaces for Superhydrophobicity, Self-Cleaning, Low Adhesion, and Drag Reduction. Progress in Materials Science, 56, 1-108. https://doi.org/10.1016/j.pmatsci.2010.04.003

[2] Yan, Y.Y., Gao, N. and Barthlott, W. (2011) Mimicking Natural Superhydrophobic Surfaces and Grasping the Wetting Process: A Review on Recent Progress in Preparing Superhydrophobic Surfaces. Advances in Colloid and Interface Science, 169, 80-105. https://doi.org/10.1016/j.cis.2011.08.005

[3] Ensikat, H.J., Ditsche-Kuru, P., Neinhuis, C. and Barthlott, W. (2011) Superhydrophobicity in Perfection: The Outstanding Properties of the Lotus Leaf. Beilstein Journal of Nanotechnology, 2, 152-161. https://doi.org/10.3762/bjnano.2.19

[4] Fang, Y., et al. (2008) A Study on the Forms and Self-Cleaning Mechanism of the Scales on Butterfly Wing Surfaces. PhD Thesis, Jilin University, Changchun.

[5] Feng, L., Zhang, Y.A., Xi, J.M., Zhu, Y., Wang, N., Xia, F. and Jiang, L. (2008) Petal Effect: A Superhydrophobic State with High Adhesive Force. Langmuir, 24, 4114-4119. https://doi.org/10.1021/la703821h

[6] Wang, M.C., Yang, Q.L., Wang, C., Wang, J.M. and Jiang, L. (2011) Relationship between Rose Petal Microstructure and Water Droplet Adhesion. Chemical Journal of Chinese University, 32, 1594-1597.

[7] Zheng, Y.M., Gao, X.F. and Jiang, L. (2007) Directional Adhesion of Superhydrophobic Butterfly Wings. Soft Matter, 3, 178-182. https://doi.org/10.1039/B612667G

[8] Yu, M.D., et al. (2019) Fabrication of Durable and Roughness Regeneration Superhydrophobic Composite Materials by Hot Pressing. Composites Part B, Engineering, 179, Article ID: 107431. https://doi.org/10.1016/j.compositesb.2019.107431

[9] Wang, F.P., et al. (2017) A Lotus-Leaf-Like $\mathrm{SiO}_{2}$ Superhydrophobic Bamboo Surface Based on Soft Lithography. Colloids and Surfaces A: Physicochem, 520, 834-840. https://doi.org/10.1016/j.colsurfa.2017.02.043

[10] Yang, J., et al. (2017) Preparation, Characterization, and Properties of Fluorine-Free Superhydrophobic Paper Based on Layer-by-Layer Assembly. Carbohydrate Polymers, 178, 228-237. https://doi.org/10.1016/j.carbpol.2017.09.040

[11] Yan, Y.Y., et al. (2011) Mimicking Natural Superhydrophobic Surfaces and Grasping the Wetting Process: A Review on Recent Progress in Preparing Superhydrophobic Surfaces. Advances in Colloid and Interface Science, 169, 80-105. https://doi.org/10.1016/j.cis.2011.08.005

[12] Yang, M.P., et al. (2018) Fabrication of Superhydrophobic Cotton Fabric with Fluorinated $\mathrm{TiO}_{2}$ Sol by a Green and One-Step Sol-Gel Process. Carbohydrate Polymers, 197, 75-82. https://doi.org/10.1016/j.carbpol.2018.05.075

[13] Shirtcliffe, N.J., et al. (2007) Superhydrophobic to Superhydrophilic Transitions of Sol-Gel Films for Temperature, Alcohol or Surfactant Measurement. Materials Chemistry and Physics, 103, 112-117. 
[14] Ni, L.L., et al. (2019) Light-Induced Crystallization-Driven Formation of Hierarchically Ordered Superhydrophobic Sol-Gel Coatings. Progress in Organic Coatings, 135, 255-262. https://doi.org/10.1016/j.porgcoat.2019.05.045

[15] Zhong, X.-M., et al. (2016) Preparation of Robust Anti-Smudge Coatings via Electrophoretic Deposition. Chemical Engineering Journal, 302, 744-755. https://doi.org/10.1016/j.cej.2016.05.112

[16] Jia, W., et al. (2019) Superhydrophobic Membrane by Hierarchically Structured PDMS-POSS Electrospray Coating with Cauliflower-Shaped Beads for Enhanced MD Pergormance. Journal of Membrane Science, 597, Article ID: 117638. https://doi.org/10.1016/j.memsci.2019.117638

[17] Liu, Y.B., et al. (2019) Desigh and Preparation of Biomimetic Polydimethylsiloxane Films with Superhydrophobic, Selfhealing and Drag Reduction Properties via Replication of Shark Skin and SI-ATRP. Chemical Engineering Journal, 356, 318-328. https://doi.org/10.1016/j.cej.2018.09.022

[18] Zhang, Q.L., et al. (2019) Enhanced Dielectric and Hydrophobic Properties of PDMS/P (VDF-TrFE) Blend Films by Embedding PS Microspheres. Colloids and surfaces $A, 569,171-178$. https://doi.org/10.1016/j.colsurfa.2019.03.006

[19] Sarkar, D.K., et al. (2008) Superhydrophobic Properties of Ultrathin rf-Sputtered Teflon Films Coated Etched Aluminum Surfaces. Materials Letters, 62, 1226-1229. https://doi.org/10.1016/j.matlet.2007.08.051

[20] Zhu, X., et al. (2020) Perfluorinated Superhydrophobic and Oleophobic SiO $@$ @PTFE Nanofiber Membrane with Hierarchical Nanostructures for Oily Fume Purification. Journal of Membrane Science, 594, Article ID: 117473. https://doi.org/10.1016/j.memsci.2019.117473

[21] Xu, L.B., et al. (2005) Reversible Conversion of Conducting Polymer Films from Superhydrophobic to Superhydrophilic. Angewandte Chemie, 117, 6163-6166. https://doi.org/10.1002/ange.200500868

[22] Cheng, Y.Y., et al. (2008) Facile Preparation of High Density Polyethylene Superhydrophobic/Superoleophilic Coatings on Glass, Copper and Polyurethane Sponge for Self-Cleaning, Corrosion Resistance and Efficient Oil/Water Separation. Journal of Colloid and Interface Science, 525, 76-85. https://doi.org/10.1016/j.jcis.2018.04.075

[23] Liang, J.Y., et al. (2016) $\mathrm{SiO}_{2}$-g-PS/Fluoroalkylsilane Composites for Superhydrophobic and Highly Oleophobic Coatings. Colloids and Surfaces A: Physicochemical and Engineering Aspects, 507, 26-35. https://doi.org/10.1016/j.colsurfa.2016.07.056

[24] Zhang, N., et al. (2018) Dual-Templating Synthesis of Compressible and Superhydrophobic Spongy Polystyrene for Oil Capture. Chemical Engineering Journal, 154, 245-253. https://doi.org/10.1016/j.cej.2018.07.184

[25] Moatmed, S.M., et al. (2019) Highly Efficient and Reusable Superhydrophobic/Superoleophilic Polystyrene@ $\mathrm{Fe}_{3} \mathrm{O}_{4}$ Nanofiber Membrane for High-Performance Oil/Water Separation. Journal of Environmental Chemical Engineering, 7, Article ID: 103508. https://doi.org/10.1016/j.jece.2019.103508

[26] Cheng, Y.Y., et al. (2017) Controlled Fabrication of $\mathrm{NiO} / \mathrm{ZnO}$ Superhydrophobic Surface on Zinc Substrate with Corrosion and Abrasion Resistance. Journal of Alloys and Compounds, 723, 225-236. https://doi.org/10.1016/j.jallcom.2017.06.241

[27] He, G., et al. (2018) Durable Superhydrophobic $\mathrm{Zn} / \mathrm{Ano} / \mathrm{TiO}_{2}$ Surfaces on Ti6Al4V Substrate with Self-Cleaning Property and Switchable Wettability. Ceramics International, 44, 638-647. https://doi.org/10.1016/j.ceramint.2017.09.223 
[28] Bharathidasan, T., et al. (2014) Effect of Wettability Surface Roughness on Ice-Adhesion Strength of Hydrophilic, Hydrophobic and Superhydrophobic Surfaces. Applied Surface Science, 314, 241-250. https://doi.org/10.1016/j.apsusc.2014.06.101

[29] Subramanyam, S.B., et al. (2016) Low Ice Adhesion on Nano-Textured Superhydrophobic Surfaces under Supersaturated Conditions. ACS Applied Materials \& Interfaces, 8, 12583-12587. https://doi.org/10.1021/acsami.6b01133

[30] Li, X.L., et al. (2019) Fabrication of Bio-Inspired Non-Fluorinated Superhydrophobic Surfaces with Anti-Icing Property and Its Wettability Transformation Analysis. Applied Surface Science, 505, Article ID: 144386.

https://doi.org/10.1016/j.apsusc.2019.144386

[31] Mobarakeh, F., Jafari, R. and Farzaneh, M. (2013) The Ice Repellency of Plasma Polymerized Hexamethyldisiloxane Coating. Applied Surface Science, 284, Article ID: 45963. https://doi.org/10.1016/j.apsusc.2013.07.119

[32] Liu, E., Yin, X., Hu, J., Yu, S., Zhao, Y. and Xiong, W. (2019) Fabrication of a Biomimetic Hierarchical Superhydrophobic Cu-Ni Coating with Self-Cleaning and Anti-Corrosion Properties. Colloids and Surfaces A: Physicochemical and Engineering Aspects, 586, Article ID: 124223. https://doi.org/10.1016/j.colsurfa.2019.124223

[33] Zhang, B.B., Zhu, Q.J., Li, Y.T. and Hou, B.R. (2018) Facile Fluorine-Free One Step Fabrication of Superhydrophobic Aluminum Surface towards Self-Cleaning and Marine Anticorrosion. Journal of Chemical Engineering, 352, 625-635. https://doi.org/10.1016/j.cej.2018.07.074

[34] Xu, C.L., Song, F., Wang, X.L. and Wang, Y.Z. (2017) Surface Modification with Hierarchical $\mathrm{CuO}$ Arrays toward a Flexible, Durable Superhydrophobic and Self-Cleaning Material. Journal of Chemical Engineering, 313, 1328-1334. https://doi.org/10.1016/j.cej.2016.11.024

[35] Katerina, T., Panagiota, S.P., Dimitris, P.P. and Sotirios, E.K. (2012) Controlled Protein Adsorption on Microfluidic Channels with Engineered Roughness and Wettability. Sensors and Actuators B, 161, 216-222. https://doi.org/10.1016/j.snb.2011.10.022

[36] Zhang, B.B., Guan, F., Zhao, X., Zhang, Y.M. and Li, Y.T. (2019) Icro-Nano Textured Superhydrophobic 5083 Aluminum Alloy as a Barrier against Marine Corrosion and Sulfate-Reducing Bacteria Adhesion. Journal of the Taiwan Institute of Chemical Engineers, 97, 433-440. https://doi.org/10.1016/j.jtice.2019.01.031

[37] Shen, I., et al. (2012) Asymmetric Free-Standing Film with Multifunctional Anti-Bacterial and Self-Cleaning Properties. ACS Applied Materials \& Interfaces, 4, Article ID: 447683. https://doi.org/10.1021/am301118f

[38] Milionis, A., Loth, E. and Bayer, I.S. (2016) Recent Advances in the Mechanical Durability of Superhydrophobic Materials. Advances in Colloid and Interface Science, 229, 57-79. https://doi.org/10.1016/j.cis.2015.12.007

[39] Webb, H.K., Crawford, R.J. and Ivanova, E.P. (2014) Wettability of Natural Superhydrophobic Surfaces. Advances in Colloid and Interface Science, 210, 58-64. https://doi.org/10.1016/j.cis.2014.01.020

[40] Falde, E.J., Yohe, S.T., Colson, Y.L. and Grinstaff, M.W. (2016) Superhydrophobic Materials for Biomedical Applications. Biomaterials, 104, 87-103. https://doi.org/10.1016/j.biomaterials.2016.06.050 